\title{
HARNACK ESTIMATES AND EXTINCTION PROFILE FOR WEAK SOLUTIONS OF CERTAIN SINGULAR PARABOLIC EQUATIONS
}

\author{
E. DIBENEDETTO AND Y. C. KWONG
}

\begin{abstract}
We establish an intrinsic Harnack estimate for nonnegative weak solutions of the singular equation (1.1) below, for $m$ in the optimal range $\left((N-2)_{+} / N, 1\right)$. Intrinsic means that, due to the singularity, the space-time dimensions in the parabolic geometry must be rescaled by a factor determined by the solution itself. Consequences are, sharp supestimates on the solutions and decay rates as $t$ approaches the extinction time. Analogous results are shown for $p$-laplacian type equations.
\end{abstract}

\section{INTRODUCTION}

We will consider nonnegative locally bounded weak solutions of singular parabolic equations of the type

$$
\begin{aligned}
& u_{t}-\Delta u^{m}=0, \quad 0<m<1, \text { in } \mathscr{D}^{\prime}\left(\Omega_{T}\right), \\
& u \in C\left(0, T ; L_{\mathrm{loc}}^{2}(\Omega)\right), \quad u^{m} \in L^{2}\left(0, T ; W_{\mathrm{loc}}^{1,2}(\Omega)\right),
\end{aligned}
$$

and of the type

$$
\begin{aligned}
& u_{t}-\operatorname{div}\left(|D u|^{p-2} D u\right)=0, \quad 1<p<2, \\
& u \in C\left(0, T ; L_{\mathrm{loc}}^{2}(\Omega)\right) \cap L^{p}\left(0, T ; W_{\mathrm{loc}}^{1, p}(\Omega)\right) .
\end{aligned}
$$

Here $\Omega$ is an open set of $\mathbf{R}^{N}, N \geq 1,0<T<\infty, \Omega_{T} \equiv \Omega \times(0, T)$, and $D \equiv\left(\partial / \partial x_{1}, \ldots, \partial / \partial x_{N}\right)$. The notion of local weak solution (subsolution, supersolution) in the specified classes is standard and we refer to $[6,10,13]$.

For these solutions we prove an intrinsic Harnack inequality, within the ranges $(N-2)_{+} / N<m<1$ for (1.1), and $2 N /(N+1)<p<2$ for (1.2), and show that such ranges are optimal for a Harnack estimate to hold. Our estimates are independent of local supbounds.

In addition we derive $L_{\text {loc }}^{\infty}$ estimates of the solutions over a compact set $\mathscr{K} \subset \Omega_{T}$ in terms of their $L^{r}\left(\mathscr{K}^{\prime}\right)$-norm, over a larger set $\mathscr{K}^{\prime} \subset \Omega_{T}$, where

$$
r>\max \left\{1 ; \frac{N(2-p)}{p}\right\} \text { for }(1.2), \quad r>\max \left\{1 ; \frac{N(1-m)}{2}\right\} \text { for (1.1). }
$$

We show that such an $r$ is optimal for a supbound to hold. This is in contrast with the heat equation $(m=1$ in (1.1) or $p=2$ in (1.2)) where bounds on the

Received by the editors January 30, 1990.

1980 Mathematics Subject Classification (1985 Revision). Primary 35K55, 35K65, 35K99.

Partially supported by NSF grant DMS-8802883. 
solutions over $\mathscr{K}$, hold in terms of their $L^{r}\left(\mathscr{K}^{\prime}\right)$-norm for every $r>0$ (see Moser [17]).

The main results are stated in $\S 2$. Section 3 contains the statement and proofs of the $L_{\mathrm{loc}}^{\infty}$-estimates as well as comments on the Cauchy problem and the extinction rate. The remainder of the paper is devoted to the proof of the Harnack inequality.

\section{THE MAIN RESUlts}

The classical Harnack estimate for nonnegative solutions of the heat equation (see Hadamard [9], Pini [21], Moser [17]), fails to hold for solutions of (1.2) if $p \neq 2$ (see [6,7] for counterexamples). However, when $p>2$ an intrinsic version takes its place, which reduces to the classical one when $p=2$. The main idea is to work with a time scale that "measures," the degeneracy of (1.2) (see [7]). The singular case $1<p<2$ presented unsuspected difficulties and is left open.

By contrast, in the elliptic case the classical Harnack estimate holds for all $p>1$ (see Serrin [22], Trudinger [24]) and the theory is fairly complete. Accordingly, a second motivation of this work is to complete the corresponding parabolic theory by proving a Harnack-type estimate for nonnegative solutions of (1.1) and (1.2). We refer to [7] for further comments and a perspective on this issue.

Equation (1.1) arises in plasma-physics $[4,5,18]$ and it is referred to as the fast-diffusion case $(0<m<1)$ of the porous medium equation $(m>1)$.

There is an analogy between (1.1) and (1.2) both in terms of results (regularity $[6,8,13,15]$, extinction time $[3,6,13]$, growth conditions $[8,20])$ and techniques of proof. For example, when $m>1$, the theory of an intrinsic Harnack inequality can be developed for (1.1) paralleling that of (1.2) for $p>2$ (see [7]).

In this note we will work mainly with (1.1) and only briefly indicate how to modify statements and proofs for the case (1.2).

2-(I). The intrinsic Harnack inequality. Let $u$ be a locally bounded, nonnegative local weak solution of $(1.1)$ in $\Omega_{T}$. Fix $P_{0} \equiv\left(x_{0}, t_{0}\right) \in \Omega_{T}$ and assume $u\left(P_{0}\right)>0$. For $R>0$ we construct the cylinder

(2.1) $Q_{R}\left(u\left(P_{0}\right)\right) \equiv\left\{\left|x-x_{0}\right|<R\right\} \times\left\{t_{0}-\left[u\left(P_{0}\right)\right]^{1-m} R^{2}, t_{0}+\left[u\left(P_{0}\right)\right]^{1-m} R^{2}\right\}$.

By the results of [6], $u$ is locally Hölder continuous in $\Omega_{T}$ so that $u\left(P_{0}\right)$ is well defined.

Theorem 2.1. Assume that

$$
\kappa \equiv N(m-1)+2>0 .
$$

Then there exist constants $\delta \in(0,1)$ and $C>1$ depending only upon $N, m$ such that

$$
\begin{aligned}
& \forall P_{0} \in \Omega_{T}, \quad \forall R>0 \quad \text { such that } Q_{4 R}\left(u\left(P_{0}\right)\right) \subset \Omega_{T} ; \\
& u\left(P_{0}\right) \leq C \inf _{x \in B_{R}} u\left(x, t_{0}+\theta\right) ; \quad \theta=\delta\left[u\left(P_{0}\right)\right]^{1-m} R^{2} .
\end{aligned}
$$

Remark 2.1. The proof shows that the constants $C^{-1}, \delta$ degenerate as $N(m-1)+2 \rightarrow 0$ (i.e., $\delta, C^{-1}(N, m) \rightarrow 0$ as $\left.\kappa \rightarrow 0\right)$. However, they are "stable" as $m \rightarrow 1$, i.e., $\delta(N, m), C^{-1}(N, m) \rightarrow \delta(N, 1), C^{-1}(N, 1) \in(0,1)$ 


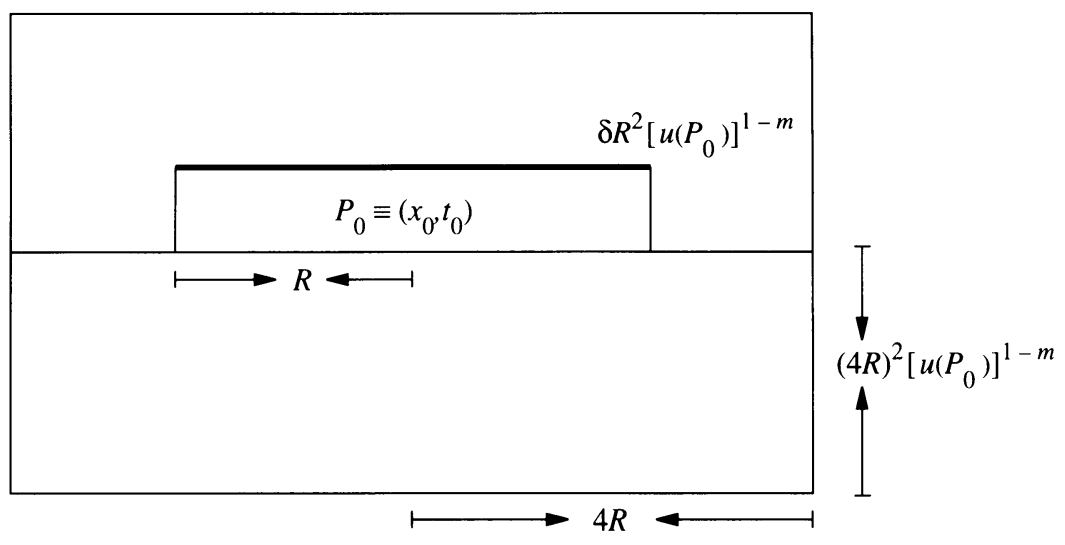

FIGURE 1

as $m \rightarrow 1$. Therefore letting $m \rightarrow 1$ in (2.3) we recover the classical inequality of Hadamard [9] and Pini [21] for nonnegative solutions of the heat equation, in the form of Krylov-Safonov [12]. See Figure 1.

Condition (2.2) is equivalent to the range of $m$

$$
\frac{(N-2)_{+}}{N}<m<1 .
$$

Remark 2.2. Such a range is optimal for (2.3) to hold, as shown by the following argument. Solutions of the Cauchy problem

$$
\left\{\begin{array}{l}
u_{t}-\Delta u^{m}=0 \quad \text { in } \mathbf{R}^{N} \times(0, \infty), \\
u(\cdot, 0)=u_{0} \in L^{1}\left(\mathbf{R}^{N}\right) \cap L^{N(1-m) / 2}\left(\mathbf{R}^{N}\right), \quad u_{0} \geq 0,
\end{array}\right.
$$

become extinct after a finite time $T^{*}$, if $\kappa \equiv N(m-1)+2<0$, (see BénilanCrandall [3]); i.e., there exists a time $T^{*}$ depending only upon $N, m$ and $\left\|u_{0}\right\|_{N(1-m) / 2, \mathbf{R}^{N}}$ such that

$$
u(x, t)=0, \quad \forall t \geq T^{*}, \forall x \in \mathbf{R}^{N},
$$

and $u(\cdot, t)$ is not identically zero for $t<T^{*}$. The extinction profile, i.e., the boundary of the set $[u>0] \cap\{t>0\}$ is the hyperplane $t=T^{*}$ (see [6]). That is, $u(x, t)>0, \forall x \in \mathbf{R}^{N}, \forall t<T^{*}$ (see also Kwong [13] for a similar result in bounded domains).

It was also shown in [3] that extinction cannot occur in finite time if $\kappa \geq 0$.

If (2.3) were to hold for $m<(N-2)_{+} / N$, we could fix $\left(x_{0}, t_{0}\right)$ so that $T^{*}-t_{0}=\varepsilon \in(0,1)$ is arbitrarily small and choose $R$ so large that

$$
\delta u^{1-m}\left(x_{0}, t_{0}\right) R^{2}=\varepsilon,
$$

to get a contradiction.

When $\Omega$ is bounded and $\partial \Omega$ smooth, solutions of (1.1) with data $\left.u\right|_{\partial \Omega}=0$ and $u(\cdot, 0)=u_{0} \in L^{\infty}(\Omega)$ exhibit extinction in finite time for $m$ in the whole range $0<m<1$ (see [3] and references therein). In such a case however $R$ cannot be taken arbitrarily large.

If there is a finite extinction time $T^{*}$, then $u(\cdot, t)$ decays to zero as $t \rightarrow T^{*}$ at the rate $\left(T^{*}-t\right)^{1 /(1-m)}($ see $\S 3-(\mathrm{II}))$. 
Similar results hold for the $p$-laplacian equation (1.2). Let $u$ be a locally bounded nonnegative local weak solution of (1.2) in $\Omega_{T}$. Fix $P_{0} \equiv\left(x_{0}, t_{0}\right) \in$ $\Omega_{T}$, assume $u\left(P_{0}\right)>0$ and for $R>0$ consider the box

$$
Q_{R}\left(u\left(P_{0}\right)\right) \equiv\left\{\left|x-x_{0}\right|<R\right\} \times\left\{t_{0}-\left[u\left(P_{0}\right)\right]^{2-p} R^{p}, t_{0}+\left[u\left(P_{0}\right)\right]^{2-p} R^{p}\right\} .
$$

It follows from [6] that $u\left(P_{0}\right)$ is well defined.

Theorem 2.2. Assume that

$$
\bar{\kappa} \equiv N(p-2)+p>0 .
$$

There exist constants $\delta \in(0,1), C>1$ depending only upon $N, p$ such that

$$
\begin{aligned}
& \forall P_{0} \in \Omega_{T}, \quad \forall R>0 \text { such that } Q_{4 R}\left(u\left(P_{0}\right)\right) \subset \Omega_{T}, \\
& u\left(P_{0}\right) \leq C \inf _{x \in B_{R}} u\left(x, t_{0}+\theta\right), \quad \theta=t_{0}+\delta\left[u\left(P_{0}\right)\right]^{2-p} R^{p} .
\end{aligned}
$$

The constants $C^{-1}$ and $\delta \rightarrow 0$ as $p \rightarrow 2 N /(N+1)$ but are "stable" as $p \nearrow 2$. Also, (2.7) cannot hold if $p<2 N /(N+2)$ i.e., $\bar{\kappa}<0$.

The last statement can be proved as before by making use of the finite-time extinction property of solutions of the Cauchy problem associated with (1.2) with initial data $u_{0} \in L^{1}\left(\mathbf{R}^{N}\right) \cap L^{N(2-p) / p}\left(\mathbf{R}^{N}\right)$. This property in turn can be proved by a simple adaptation of the arguments of Benilan-Crandall [3] (see also Herrero-Vasquez [11]).

The proofs of Theorems 2.1 and 2.2 are more involved than that of their "degenerate" counterpart in [7]. The two main ingredients are the local Hölder continuity of solutions and an expansion of the positivity set of the solutions "sidewise" i.e., along the space variables only.

Local Hölder continuity has been established in [6] and exploits a special space-time configuration (see $\S 5$ and Appendix A). The expansion of positivity is achieved by constructing suitable comparison functions (see $\S 4$ and Appendix B), sensitive to conditions (2.2) and (2.6).

\section{The $L_{\text {loc }}^{\infty}$-ESTIMATE}

Let $u$ be a locally bounded nonnegative local weak solution of (1.1) in $\Omega_{T}$ and consider the box

$$
Q_{R} \equiv B_{R} \times\{0, t\}, \quad B_{R} \equiv\{|x|<R\} .
$$

After a suitable translation, we may assume $Q_{4 R} \in \Omega_{T}$, provided $R$ and $t>0$ are sufficiently small.

Let $r \geq 1$ be any number satisfying

$$
\kappa_{r} \equiv N(m-1)+2 r>0 \text {. }
$$

Theorem 3.1. Let $r \geq 1$ and $0<m<1$ satisfy (3.1). There exists a constant $\gamma=\gamma(N, m, r)$ such that

$$
\forall t>0, \quad \forall R>0 \text { such that } Q_{4 R} \subset \Omega_{T}
$$

$$
\sup _{x \in B_{R}} u(x, t) \leq \gamma t^{-N / \kappa_{r}}\left(\sup _{0<\tau<t} \int_{B_{2 R}} u^{r}(x, \tau) d x\right)^{2 / \kappa_{r}}+\gamma\left(\frac{t}{R^{2}}\right)^{1 /(1-m)} \text {. }
$$


Remark 3.1. The proof shows that $u$ could be a nonnegative subsolution. Moreover the pair of balls $B_{R}, B_{2 R}$ in (3.2) could be replaced by any pair $B_{R}, B_{(1+\sigma) R}$, $\forall \sigma>0$ with $\gamma$ depending also upon $\sigma$, provided $Q_{(1+\sigma) R} \subset \Omega_{T}$.

Remark 3.2. The constant $\gamma \rightarrow \infty$ as $\kappa_{r} \equiv N(m-1)+2 r \rightarrow 0$. However it is stable as $m \rightarrow 1$. The condition $\kappa_{r}>0$ is optimal for a supestimate to hold (see $\S 3$-(III) below).

The theorem turns the qualitative information $u \in L_{\mathrm{loc}}^{\infty}\left(\Omega_{T}\right)$ into the quantitative estimate (3.2). In particular solutions that can be constructed as $L_{\mathrm{loc}}^{r}\left(\Omega_{T}\right)$ weak limits of smooth approximations, satisfy (3.2).

In the next theorem, (3.4) holds for nonnegative weak solutions not necessarily bounded, and $\forall 0<m<1$. Also (3.3) holds $\forall r \geq 1$.

Theorem 3.2. $\exists \gamma=\gamma(N, m, r)$ such that

$$
\forall 0<t<\infty, \quad \forall R>0 \text { such that } Q_{4 R} \subset \Omega_{T},
$$

$$
\begin{aligned}
& \sup _{0<\tau<t} \int_{B_{R}} u^{r}(x, \tau) d x \leq \gamma\left\{\int_{B_{2 R}} u^{r}(x, 0) d x+\left(\frac{t^{r}}{R^{\kappa_{r}}}\right)^{1 /(1-m)}\right\}, \\
& \forall 0<t<\infty, \quad \forall R>0 \text { such that } Q \subset \Omega_{T}, \\
& \sup _{0<\tau<t} \int_{B_{R}} u(x, \tau) d x \leq \gamma\left\{\int_{B_{2 R}} u(x, t) d x+\left(\frac{t}{R^{\kappa}}\right)^{1 /(1-m)}\right\},
\end{aligned}
$$

where $\kappa \equiv \kappa_{1} \equiv N(m-1)+2>0$.

Remark 3.3. The proof shows that the constant $\gamma \rightarrow \infty$ as $m \rightarrow 1$.

3-(I). $L^{\infty}$-estimates at the same time level. Of particular interest for the proof of the Harnack inequality is the following supestimate obtained by combining Theorem 3.1 and 3.2, under the assumptions

$$
r=1, \quad \kappa \equiv N(m-1)+2>0 .
$$

Lemma 3.3. Let (3.5) hold. There exist a constant $\gamma=\gamma(m, N)$ such that

$$
\begin{aligned}
& \forall 0<t<\infty, \quad \forall R>0 \text { such that } Q_{4 R} \subset \Omega_{T}, \\
& \sup _{x \in B_{R}} u(x, t) \leq \gamma t^{-N / \kappa}\left(\int_{B_{2 R}} u(x, t) d x d t\right)^{2 / \kappa}+\gamma\left(\frac{t}{R^{2}}\right)^{1 /(1-m)} .
\end{aligned}
$$

Remark 3.4. The interest in (3.6) is that the sup of $u$ over a ball $B_{R}$ at some level $t>0$ is estimated in terms of the $L^{1}\left(B_{4 R}\right)$-norm of $u$ at the same level $t$. It is precisely this particular feature that will make possible the proof of the Harnack inequality.

We will use this fact in the following form. Assume the cylinder

$$
Q(4 R, 4) \equiv B_{4 R} \times\{-4,0\}
$$

is all contained in the domain of definition of $u$. By Theorems 3.1 and 3.2:

$$
\begin{aligned}
& \forall-2<\tau<0, \\
& \|u(\cdot, \tau)\|_{\infty, B_{R}} \leq \gamma \sup _{-4<\tau<0}\left(\int_{B_{2 R}} u(x, \tau) d x\right)^{2 / \kappa}+\gamma\left(\frac{1}{R^{2}}\right)^{1 /(1-m)} .
\end{aligned}
$$


By (3.4) we conclude that

$$
\begin{aligned}
& \forall-2<\tau<0, \\
& \|u(\cdot, \tau)\|_{\infty, B_{R}} \leq \gamma\left(\int_{B_{4 R}} u(x, 0) d x\right)^{2 / \kappa}+\gamma R^{2 /(m-1)} .
\end{aligned}
$$

Remark 3.5. The constant $\gamma$ depends only upon $N$ and $m$. It follows from Remarks 3.2 and 3.3 that $\gamma \rightarrow \infty$ as either $\kappa \rightarrow 0$ or $m \rightarrow 1$.

3-(II). Rate of extinction. Suppose $u$ is a nonnegative local weak solution of (1.1) in some domain contained in $\Omega \times \mathbf{R}^{+}$, and assume there exists a finite extinction time $T^{*}$. This occurs for all $0<m<1$ in bounded domains and for $N(m-1)+2<0$ in the whole $\mathbf{R}^{N}$ (see [3]).

Applying (3.4) to $u$ within the box

$$
Q\left(T^{*}\right) \equiv B_{4 R} \times\left(T^{*}-t, T^{*}\right) \subset \Omega \times \mathbf{R}^{+},
$$

we obtain

$$
\int_{B_{R}} u(x, t) d x \leq \gamma\left(\frac{T^{*}-t}{R^{\kappa}}\right)^{1 /(1-m)} .
$$

Thus the $L_{\mathrm{loc}}^{1}(\Omega)$ norm of $u(\cdot, t)$ decays to zero as $t \rightarrow T^{*}$ at the rate $\left(T^{*}-t\right)^{1 /(1-m)}$.

If in addition $\kappa \equiv N(m-1)+2>0$, by combining the sup estimate of Theorem 3.1 with (3.4) we have

Corollary. Let $N(m-1)+2>0$ and assume $T^{*}>0$ is a finite extinction time for $u$. Then $\exists \gamma=\gamma(N, m)$ such that

$$
\begin{aligned}
& \forall R>0, \quad \forall t \text { such that } B_{4 R} \times\left(2 t-T^{*}, T^{*}\right) \subset \Omega \times \mathbf{R}^{+}, \\
& \|u(\cdot, t)\|_{\infty, B_{R}} \leq \gamma\left(\frac{T^{*}-t}{R^{2}}\right)^{1 /(1-m)} .
\end{aligned}
$$

3-(III). The Cauchy problem with data in $L_{\text {loc }}^{r}\left(\mathbf{R}^{N}\right)$. We may combine Theorems 3.1 and 3.2 to deduce existence of locally Hölder continuous solutions for the Cauchy problem.

Consider the Cauchy problem

$$
\begin{cases}u_{t}-\Delta u^{m}=0 & \text { in } S_{T} \equiv \mathbf{R}^{N} \times(0, T) \\ u(\cdot, 0)=u_{0}(x) \in L_{\mathrm{loc}}^{r}\left(\mathbf{R}^{N}\right), & u_{0} \geq 0 \\ 0<T<\infty, & r \geq 1\end{cases}
$$

If $r \geq 1$ satisfies (3.1) we may construct the solution by approximating $u_{0}$, in $L_{\text {loc }}^{r}\left(\mathbf{R}^{N}\right)$, with $u_{0, n} \in C_{0}^{\infty}\left(\mathbf{R}^{N}\right), n \in \mathbf{N}$ satisfying

$$
\int_{B_{R}} u_{0, n}^{r} d x \leq \gamma \int_{B_{R}} u_{0}^{r} d x, \quad \forall R>1, \quad n=0,1, \ldots,
$$

for some constant $\gamma=\gamma(m, N)$. Then if $(x, t) \rightarrow u_{n}(x, t)$ are the corresponding solutions of (3.18) with $u_{0}$ replaced by $u_{0, n}$, we deduce from Theorems 3.1 and 3.2 that $\exists \gamma=\gamma(m, N)$, such that

$$
\begin{aligned}
& \forall t>0, \quad \forall R>0, \quad \forall n \in \mathbf{N}, \\
& \sup _{x \in B_{R}} u_{n}(x, t) \leq \gamma t^{-N / \kappa_{r}}\left(\int_{B_{2 R}} u_{0}^{r} d x\right)^{2 / \kappa_{r}}+\gamma\left(\frac{t}{R^{2}}\right)^{1 /(1-m)} .
\end{aligned}
$$


Thus $\left\{u_{n}\right\}$ are equi-bounded over compact subsets of $\mathbf{R}^{N} \times(0, \infty)$ and by the results of [6] are equi-Hölder continuous. This suffices to prove existence of solutions. The initial datum is taken in the sense of $L_{\text {loc }}^{r}\left(\mathbf{R}^{N}\right)$. Uniqueness can be shown by a technique similar to the one in $[8,10]$.

Remark 3.6. When $u_{0} \in L_{\text {loc }}^{1}\left(\mathbf{R}^{N}\right)$ existence and uniqueness of solutions is due to Herrero-Pierre [10]. The point here is to show that a precise local integrability of the initial datum $u_{0}$ (i.e., $u_{0} \in L_{\mathrm{loc}}^{r}\left(\mathbf{R}^{N}\right)$ ) yields locally bounded and locally Hölder-continuous solutions.

The order of integrability of $u_{0}$ to ensure a $C_{\text {loc }}^{\alpha}$-solution is sharp as shown by the following example.

Consider the function

$$
x \rightarrow g(x) \equiv \frac{\left(\varepsilon^{2}-|x|^{2}\right)_{+}^{a_{1}}}{\left.\left.|x|^{N}|\ln | x\right|^{2}\right|^{a_{2}}}, \quad \varepsilon \in(0,1), a_{1}, a_{2}>1,
$$

and set

$$
Z(x, t)=(1-h t)+g(x), \quad h>0 .
$$

We observe that if $a_{2}>1, g \in L^{1}\left(\mathbf{R}^{N}\right)$ but $g \notin L_{\text {loc }}^{r}\left(\mathbf{R}^{N}\right), \forall r>1$.

Lemma 3.3. Assume $m=(N-2) / N, N \geq 3$. The constants $\varepsilon \in(0,1), a_{1}, a_{2}$, $h>1$ can be chosen depending only upon $N$ so that

$$
\left\{\begin{array}{l}
Z_{t}-\Delta Z^{m} \leq 0 \quad \text { a.e. } \mathbf{R}^{N} \times(0, \infty) \\
Z(\cdot, 0)=g(\cdot)
\end{array}\right.
$$

Now the Cauchy problem associated with (1.1) and initial datum $u_{0}=g$ has a unique weak solution $u$ satisfying

$$
u \geq Z \quad \forall t \geq 0,
$$

by the comparison principle (see Herrero-Pierre [10], Pierre [20]). Thus if $t \in(0,1 / h), \liminf _{|x| \rightarrow 0} u(x, t)=\infty$.

The proof of the lemma is given in Appendix $\mathrm{C}$.

3-(IV). Proof of Theorem 3.1. Let $\sigma \in(0,1]$ be fixed and consider the sequence of radii and time levels

$$
R_{n}=R\left(1+\sigma 2^{-n}\right), \quad t_{n}=\frac{t}{2}\left(1-\sigma 2^{-n}\right) .
$$

Set

$$
B_{n} \equiv B_{R_{n}}, \quad Q_{n} \equiv B_{n} \times\left(t_{n}, t\right), \quad n=0,1,2, \ldots,
$$

and let $(x, \tau) \rightarrow \zeta_{n}(x, \tau)$ be a nonnegative piecewise smooth function in $Q_{n}$ that equals one on $Q_{n+1}$, vanishing on $\partial B_{n}$ and such that

$$
\left|D \zeta_{n}\right| \leq \frac{2^{n+1}}{\sigma R}, \quad 0 \leq \zeta_{n, t} \leq \frac{2^{n+2}}{\sigma t}
$$

Consider also the sequence of increasing levels

$$
k_{n}=k\left(1-\frac{1}{2^{n+1}}\right), \quad n=0,1,2, \ldots,
$$

where $k>0$ will be chosen later. 
First write (1.1) in the form

$$
\frac{\partial}{\partial t} v^{1 / m}-\Delta v=0, \quad v=u^{m} .
$$

In the weak formulation of (3.13) take testing functions $\left(v-k_{n}\right)_{+}^{q-1} \zeta_{n}^{2}, q>1$, to be chosen, and integrate by parts over $Q_{n}$. Setting

$$
w_{n}=\left(v-k_{n}\right)_{+}^{q / 2} \zeta_{n},
$$

we obtain by standard calculations

$$
\begin{array}{r}
k^{(1-m) / m} \sup _{t_{n} \leq \tau \leq t} \int_{B_{n}} w_{n}^{2}(\tau) d x+\iint_{Q_{n}}\left|D w_{n}\right|^{2} d x d \tau \\
\leq \frac{\gamma 2^{2 n}}{\sigma^{2} t}\left(\frac{t}{R^{2}}+\|u\|_{\infty, Q_{0}}^{1-m}\right) \iint_{Q_{n-1}} w_{n-1}^{2} d x d \tau .
\end{array}
$$

If $\|u\|_{\infty, Q_{0}}^{1-m}<t / R^{2}$ there is nothing to prove. Otherwise we rewrite (3.15) as

$$
\begin{gathered}
k^{(1-m) / m} \sup _{t_{n} \leq \tau \leq t} \int_{B_{n}} w_{n}^{2}(\tau) d x+\iint_{Q_{n}}\left|D w_{n}\right|^{2} d x d \tau \\
\leq \frac{\gamma 2^{2 n}}{\sigma^{2} t}\|u\|_{\infty, Q_{0}}^{1-m} \iint_{Q_{n-1}} w_{n-1}^{2} d x d \tau .
\end{gathered}
$$

By Hölder inequality and the space-time version of the Gagliardo-Nirenberg multiplicative inequality (see $[16$, p. 74$]$ ) we get

$$
\begin{aligned}
& \iint_{Q_{n}} w_{n}^{2} d x d \tau \leq\left(\iint_{Q_{n}} w_{n}^{2(N+2) / N} d x d \tau\right)^{N /(N+2)}\left|A_{n}\right|^{2 /(N+2)} \\
& \quad \leq\left\{\left(\sup _{l_{n} \leq \tau \leq t} \int_{B_{n}} w_{n}^{2}(\tau) d x\right)^{2 / N}\left(\iint_{Q_{n}}\left|D w_{n}\right|^{2} d x d \tau\right)\right\}^{N /(N+2)}\left|A_{n}\right|^{2 /(N+2)},
\end{aligned}
$$

where

$$
A_{n} \equiv\left\{(x, t) \in Q_{n} \mid v(x, t)>k_{n}\right\} .
$$

From this and $\left(3.15^{\prime}\right)$

$$
\iint_{Q_{n}} w_{n}^{2} d x d \tau \leq \frac{\gamma 2^{2 n}}{\sigma^{2} t}\|u\|_{\infty, Q_{0}}^{1-m} k^{-((1-m) / m) 2 /(N+2)}\left(\iint_{Q_{n-1}} w_{n-1}^{2}\right)\left|A_{n}\right|^{2 /(N+2)} .
$$

Since

$$
\iint_{Q_{n-1}} w_{n-1}^{2} d x d \tau \geq 2^{-(n+1) q} k^{q}\left|A_{n}\right|
$$

we obtain from (3.18)

$$
\iint_{Q_{n}} w_{n}^{2} d x d \tau \leq \frac{\gamma d_{0}^{n}}{\sigma^{2} t}\|u\|_{\infty, Q_{0}}^{1-m} k^{-((1-m+r) / m) 2 /(N+2)}\left(\iint_{Q_{n-1}} w_{n-1}^{2}\right)^{1+2 /(N+2)},
$$

where we have set

$$
r=m q, \quad d_{0}=4^{1+q /(N+2)} .
$$


From the recursive inequalities (3.19) it follows by virtue of Lemma 5.7 of [16, p. 96], that $\iint_{Q_{n}} w_{n}^{2} d x d \tau \rightarrow 0$ as $n \rightarrow \infty$ provided $k$ is chosen to satisfy

$$
\iint_{Q_{0}} v^{q} d x d \tau=C \sigma^{N+2} t^{(N+2) / 2} k^{(1-m+r) / m}\|u\|_{\infty, Q_{0}}^{-(1-m)(N+2) / 2} .
$$

With these choices it follows that

$$
\begin{aligned}
\|u\|_{\infty}, Q_{\infty} \leq & {\left[\frac{\gamma}{\sigma^{(N+2)}} \frac{1}{t^{(N+2) / 2}}\left(\int_{0}^{t} \int_{B_{(1+\sigma) R}} u^{r}(x, \tau) d x d \tau\right)\right]^{1 /(1-m+r)} } \\
& \times\|u\|_{\infty, Q_{0}}^{(1-m)(N+2) /(2(1-m+r))} .
\end{aligned}
$$

Consider the increasing family of radii

$$
R_{s}=R \sum_{i=0}^{s} 2^{-i}, \quad s=1,2, \ldots,
$$

let $Q_{s}$ be the family of cylinders defined as before and set

$$
Y_{s}=\|u\|_{\infty, Q_{s}} .
$$

Applying (3.21) to the pair of boxes $Q_{s} \subset Q_{s+1}$ for which $\sigma=2^{-(s+1)}$, we obtain

$$
Y_{s} \leq\left[\frac{\gamma 2^{s(N+2)}}{t^{(N+2) / 2}} \int_{0}^{t} \int_{B_{2 R}} u^{r}(x, \tau) d x d \tau\right]^{1 /(1-m+r)} \cdot Y_{s+1}^{(N+2)(1-m) /\left(\kappa_{r}+(N+2)(1-m)\right)} .
$$

If $\kappa_{r}=N(m-1)+2 r>0$, by Schwarz inequality, $\forall \nu \in(0,1)$

$$
\begin{gathered}
Y_{s} \leq \nu Y_{s+1}+\gamma(N, m, r, \nu) d_{*}^{s}\left[t^{-(N+2) / 2} \int_{0}^{t} \int_{B_{2 R}} u^{r}(x, \tau) d x d \tau\right]^{2 / \kappa_{r}}, \\
d_{*}=2^{2(N+2) / \kappa_{r}} .
\end{gathered}
$$

Iteration of these inequalities yields

$$
\begin{aligned}
\|u\|_{\infty, Q_{R} \equiv} \equiv & Y_{0} \leq \nu^{s} Y_{\infty}+\gamma(N, m, r, \nu)\left[t^{-(N+2) / 2} \int_{0}^{t} \int_{B_{2 R}} u^{r}(x, \tau) d x d \tau\right]^{2 / \kappa_{r}} \\
& \times \sum_{i=0}^{s}\left(\nu d_{*}\right)^{i}, \quad s=1,2, \ldots
\end{aligned}
$$

Choose $\nu=1 /\left(2 d_{*}\right)$ so that $\sum_{i=0}^{s}\left(\nu d_{*}\right)^{i} \leq 1$ and let $s \rightarrow \infty$ to obtain

$$
\|u\|_{\infty}, Q_{R} \leq \gamma(N, m, r) \frac{1}{t^{N / \kappa_{r}}}\left(\sup _{0<\tau<t} \int_{B_{2 R}} u^{r}(x, \tau) d x\right)^{2 / \kappa_{r}} .
$$

Because of the interpolation technique $\gamma \rightarrow \infty$ as $\kappa_{r} \rightarrow 0$.

3-(V). Proof of Theorem 3.2. For $n=0,1,2, \ldots$ let

$$
R_{n}=R \sum_{i=0}^{n} 2^{-i}, \quad B_{n}=B_{R_{n}},
$$


and let $x \rightarrow \zeta_{n+1}(x)$ be a smooth cutoff function in $B_{n+1}$ that equals one on $B_{n}$ and such that

$$
\left|D \zeta_{n}\right|<\frac{2^{n+1}}{R}, \quad\left|\Delta \zeta_{n}\right| \leq \gamma \frac{2^{2 n}}{R^{2}} .
$$

By a Steklov time-averaging we can rewrite (1.1) as

$$
\frac{\partial}{\partial t} u_{h}-\Delta\left(u^{m}\right)_{h}=0 \quad \text { a.e. } \Omega_{T}
$$

where $\forall f \in L_{\text {loc }}^{1}\left(\Omega_{T}\right), h \in(0, T)$, see [16]

$$
f_{h}(x, t)=\frac{1}{h} \int_{t}^{t+h} f(x, \tau) d \tau, \quad t \in(0, T-h) .
$$

Multiply $(1.1)_{h}$ by $u_{h}^{r-1} \zeta_{n}$, integrate by parts over $B_{n+1} \times(\tau, t), \forall \tau \in(0, t)$ and let $h \rightarrow 0$ to obtain

$$
\begin{aligned}
& \frac{1}{r} \int_{B_{n+1}} u^{r}(x, t) \zeta_{n+1} d x+\frac{4 m(r-1)}{(m+r-1)^{2}} \int_{\tau}^{t} \int_{B_{n+1}}\left|D u^{(m-1+r) / 2}\right|^{2} \zeta_{n+1}^{2} d x d \tau \\
& =\frac{1}{r} \int_{B_{n+1}} u^{r}(x, \tau) \zeta_{n+1} d x+\frac{m}{(m+r-1)} \int_{\tau}^{t} \int_{B_{n+1}} u^{m+r-1} \Delta \zeta_{n+1} d x d \tau .
\end{aligned}
$$

Proof of (3.3). Set

$$
H_{n}=\sup _{0<\tau<t} \int_{B_{n}} u^{r}(x, \tau) d x, \quad H_{0}=\int_{B_{2 R}} u^{r}(x, 0) d x .
$$

Then from (3.22)

$$
\begin{aligned}
H_{n} & \leq H_{0}+\gamma \frac{2^{2 n}}{R^{2}}\left(\frac{r m}{m+r-1}\right) \int_{0}^{t}\left(\int_{B_{n+1}} u^{r}(x, s) d x\right)^{(m-1+r) / r} R^{-N(m-1) / r} d s \\
& \leq H_{0}+\gamma 2^{2 n}\left(\frac{t^{r}}{R^{\kappa_{r}}}\right)^{1 / r} H_{n+1}^{(m-1+r) / r} .
\end{aligned}
$$

Finally applying Young's inequality we get the recursive inequalities

$$
H_{n} \leq \nu H_{n+1}+\gamma(m, N, \nu) 2^{4 r n /(1-m)}\left[H_{0}+\left(\frac{t^{r}}{R^{\kappa_{r}}}\right)^{1 /(1-m)}\right], \quad \nu \in(0,1) .
$$

By choosing $\nu$ sufficiently small this implies (3.3) by an interpolation argument similar to the one above.

Proof of (3.4). From (3.22) with $r=1$

$$
H_{n} \leq \int_{B_{2 R}} u(x, t) d x+\frac{2^{2 n}}{R^{2}} \int_{0}^{t} \int_{B_{n+1}} u^{m}(x, s) d x d s .
$$

The proof is concluded by interpolation since $0<m<1$.

Remark 3.7. Inequality (3.4) is due to Herrero-Pierre [10] by a different proof. Inequality (3.3) for $r \neq 1$ seems to be new.

Because of the interpolation technique $\gamma \rightarrow \infty$ as $m \rightarrow 1$. A similar behaviour of $\gamma$ occurs in [10]. 
Remark 3.8. Results in all analogous to the ones above hold for weak solutions of the $p$-Laplacian equation (1.2) and can be proved by following the arguments of [8].

\section{LOCAL SUBSOLUTIONS}

The main feature of our proof of the Harnack inequality consists in expanding the positivity set of $u$ both in the direction of increasing times and "sidewise" in the space variables. This will be accomplished by constructing two suitable local subsolutions of (1.1). It is precisely in this construction that the range of $m$ given by

$$
\kappa \equiv N(m-1)+2>0
$$

comes into play.

Let $b, k, \mu, \theta>0$ and consider the cylindrical domain with annular cross section

$$
Q(\theta) \equiv\left\{\frac{\mu}{b} k^{m-1}<|x|^{2}<1\right\} \times\{0, \theta\}
$$

Consider the function

$$
\psi \equiv \frac{k\left(1-|x|^{\alpha}\right)_{+}^{2 / m}}{\left(1+k^{1-m} b|x|^{2} / t\right)^{1 /(1-m)}}, \quad \alpha>0 .
$$

Lemma 4.1. The constants $b, \alpha>0$ can be chosen so that

$$
\begin{aligned}
& \forall k>0, \quad \forall \mu>0, \quad \frac{\mu}{b} k^{m-1}<1, \quad \theta=\min \left\{\frac{(1-m) \mu \kappa}{4} ; k^{1-m}\right\}, \\
& \psi_{t}-\Delta \psi^{m} \leq 0 \quad \text { a.e. } Q(\theta) .
\end{aligned}
$$

Proof. Set

$$
\begin{aligned}
& \rho=|x|, \quad|z|=k^{1-m} b \frac{\rho^{2}}{t}, \quad \mathscr{F}=(1+|z|), \\
& w=\frac{k^{m}}{\mathscr{F} m /(1-m)}, \quad v=\left(1-\rho^{\alpha}\right)_{+}^{2}, \quad \psi^{m}=w v .
\end{aligned}
$$

Since $\psi^{m}$ is radial $\Delta \psi^{m}=\left(\psi^{m}\right)^{\prime \prime}+\frac{N-1}{\rho}\left(\psi^{m}\right)^{\prime}$, where the derivatives are meant with respect to $\rho$.

By direct calculation

$$
\begin{aligned}
& \left\{\begin{array}{l}
w^{\prime}=-\frac{2 m}{1-m}\left(\frac{w}{\rho}\right) \frac{|z|}{\mathscr{F}}, \\
w^{\prime \prime}=\frac{2 m}{1-m}\left(\frac{w}{\rho^{2}}\right) \frac{|z|}{\mathscr{F}}\left(\frac{2 m}{1-m} \frac{|z|}{\mathscr{F}}+1-\frac{2}{\mathscr{F}}\right) .
\end{array}\right. \\
& \left\{\begin{array}{l}
v^{\prime}=-2 \alpha \rho^{\alpha-1}\left(1-\rho^{\alpha}\right)_{+}, \\
v^{\prime \prime}=-2 \alpha(\alpha-1) \rho^{\alpha-2}\left(1-\rho^{\alpha}\right)_{+}+2 \alpha^{2} \rho^{2 \alpha-2} .
\end{array}\right.
\end{aligned}
$$

Form the expressions $\left(\psi^{m}\right)^{\prime}=w^{\prime} v+w v^{\prime}$, and $\left(\psi^{m}\right)^{\prime \prime}=w^{\prime \prime} v+2 w^{\prime} v^{\prime}+w v^{\prime \prime}$ 
using (4.5)-(4.6) and compute

$$
\begin{aligned}
\Delta \psi^{m}= & \frac{2 m}{1-m}\left(\frac{w}{\rho^{2}}\right) \frac{|z|}{\mathscr{F}} v\left\{-N+1+\frac{2 m}{1-m} \frac{|z|}{\mathscr{F}}+1-\frac{2}{\mathscr{F}}\right\} \\
& +\frac{8 m}{1-m} \alpha\left(\frac{w}{\rho^{2}}\right) \frac{|z|}{\mathscr{F}} \rho^{\alpha}\left(1-\rho^{\alpha}\right)_{+}-2 \alpha(\alpha-1)\left(\frac{w}{\rho^{2}}\right) \rho^{\alpha}\left(1-\rho^{\alpha}\right)_{+} \\
& +(N-1)\left[-2 \alpha\left(\frac{w}{\rho^{2}}\right) \rho^{\alpha}\left(1-\rho^{\alpha}\right)_{+}\right]+2 w \alpha^{2} \rho^{2 \alpha-2} .
\end{aligned}
$$

Drop the last nonnegative term and observe that the first term in brackets on the right-hand side of (4.7) equals

$$
\{\cdots\}=\left\{-N+\frac{2}{1-m} \frac{|z|}{\mathscr{F}}\right\} .
$$

We obtain

$$
\begin{aligned}
\Delta \psi^{m} \geq & \frac{2 m}{1-m}\left(\frac{w}{\rho^{2}}\right) \frac{|z|}{\mathscr{F}} v\left\{-N+\frac{2}{1-m} \frac{|z|}{\mathscr{F}}\right\} \\
& +2 \alpha\left(\frac{w}{\rho^{2}}\right) \rho^{\alpha}\left(1-\rho^{\alpha}\right)+\left\{\frac{4 m}{1-m} \frac{|z|}{\mathscr{F}}-N+2-\alpha\right\} .
\end{aligned}
$$

We impose on $|z|$ to be so large that

$$
\begin{aligned}
-N+\frac{2}{1-m} \frac{|z|}{\mathscr{F}} & =\frac{N(m-1)+2}{1-m}-\frac{2}{(1-m) \mathscr{F}} \\
& >\kappa-\frac{2}{(1-m) \mathscr{F}}>0 .
\end{aligned}
$$

Next we choose $\alpha$ so that the second term in brackets on the right-hand side of (4.8) is nonnegative. This is accomplished as follows.

$$
\begin{aligned}
\{\cdots\} & =\left(-N+\frac{2}{1-m} \frac{|z|}{\mathscr{F}}\right)+\frac{2(2 m-1)}{1-m} \frac{|z|}{\mathscr{F}}+2-\alpha \\
& \geq \frac{2(2 m-1)}{1-m} \frac{|z|}{\mathscr{F}}+2-\alpha .
\end{aligned}
$$

If $m \geq \frac{1}{2}$ we take $\alpha=2$. If $0<m<\frac{1}{2}$ we estimate

$$
\{\cdots\} \geq-2+\frac{2 m}{1-m}+2-\alpha,
$$

and we take $\alpha=2 m /(1-m)$.

Combining these calculations we have

$$
\Delta \psi^{m} \geq \frac{2 m}{1-m}\left(\frac{w}{\rho^{2}}\right) \frac{|z|}{\mathscr{F}} v\left\{-N+\frac{2}{1-m} \frac{|z|}{\mathscr{F}}\right\},
$$

for all $|z|$ so large that (4.9) holds. Next calculate

$$
\psi_{t}=\frac{k\left(1-\rho^{\alpha}\right)_{+}^{2 / m}}{(1-m) \mathscr{F} 1 /(1-m)+1} \frac{|z|}{t} \leq \frac{w^{1 / m}}{1-m} v \frac{|z|}{\mathscr{F}} \frac{1}{t},
$$


and combine with (4.10) to obtain

(4.11)

$$
\begin{aligned}
\mathscr{L}^{*}(\psi) & \equiv\left[\psi_{t}-\Delta \psi^{m}\right] \frac{\mathscr{F} \rho^{2}(1-m)}{|z| w v} \\
& \leq w^{(1-m) / m} \frac{\rho^{2}}{t}-2 m\left(-N+\frac{2}{1-m} \frac{|z|}{\mathscr{F}}\right) \leq \frac{1}{b}-2 m\left(\kappa-\frac{2}{(1-m) \mathscr{F}}\right),
\end{aligned}
$$

since

$$
w^{(1-m) / m} \frac{\rho^{2}}{t}=\frac{k^{1-m} b \rho^{2} / t}{b \mathscr{F}}=\frac{1}{b} \frac{|z|}{|z|+1} \leq \frac{1}{b} .
$$

From the definition of the cylindrical domain $Q(\theta)$ in (4.2), $|z|=k^{1-m} b|x|^{2} / t$ $\geq \mu / t$ so that $1 / \mathscr{F} \leq t / \mu$. These remarks in (4.11) give

$$
\mathscr{L}^{*}(\psi) \leq \frac{1}{b}+\frac{4 m t}{(1-m) \mu}-2 m \kappa .
$$

To prove the lemma we choose

$$
\theta=\frac{\mu \kappa(1-m)}{4}, \quad b=\frac{1}{m \kappa} .
$$

4-(I). Scaling and comparison. The constants $b>1, \alpha>0$ being fixed, the functions

$$
\psi_{\lambda}\left(x-x_{*} ; t-t_{*}\right)=\frac{k\left(\lambda^{\alpha}-\left|x-x_{*}\right|^{\alpha}\right)_{+}^{2 / m} \lambda^{-2 \alpha / m}}{\left(1+k^{1-m} b\left|x-x_{*}\right|^{2} /\left(t-t_{*}\right)\right)^{1 /(1-m)}}, \quad \lambda>0,
$$

are subsolutions of (1.1) in the annular cylindrical domain

$$
Q_{*}^{(\lambda)} \equiv\left\{\frac{\lambda^{2} \mu k^{m-1}}{b}<\left|x-x_{*}\right|^{2}<\lambda^{2}\right\} \times\left\{t_{*}, t_{*}+\lambda^{2} \theta\right\},
$$

provided $\mu$ is chosen to satisfy

$$
\mu k^{m-1} / b<1
$$

On the parabolic boundary of $Q_{*}^{(\lambda)}$ we have

(i) For $\left|x-x_{*}\right|^{2}=\lambda^{2} \mu k^{m-1} / b, t_{*}<t<t_{*}+\lambda^{2} \theta, \psi_{\lambda}\left(x-x_{*} t-t_{*}\right) \leq k$,

(ii) For $\left|x-x_{*}\right|^{2}=\lambda^{2}, t_{*} \leq t \leq t_{*}+\lambda^{2} \theta, \psi_{\lambda}=0$,

(iii) For $t=t_{*}, \psi_{\lambda}=0$.

Thus if $u$ solves (1.1) in a domain containing $Q_{*}^{(\lambda)}$ and if

$$
u(x, t) \geq k, \quad\left|x-x_{*}\right|^{2}=\frac{\lambda^{2} \mu k^{m-1}}{b}, \quad t_{*}<t<t_{*}+\lambda^{2} \theta,
$$

then by the comparison principle

$$
u \geq \psi_{\lambda} \text { in } Q_{*}^{(\lambda)} .
$$

For remarks on the comparison principles see Appendix D.

4-(II). Time expansion of positivity. The next subsolution of (1.1) will be employed to expand the positivity set of $u$ in the direction of positive $t$.

Let $\rho_{0}>0, k>0$, set

$$
S(t)=\left(\frac{m t}{k^{1-m}}+\rho_{0}^{2}\right), \quad t \geq 0
$$


and consider the function

$$
\varphi(x, t)=\frac{k \rho_{0}^{2 \xi / m}}{S(t)^{\xi / m}}\left(1-\frac{|x|^{2}}{S(t)}\right)_{+}^{\beta / m}
$$

where $\beta, \xi>1$.

The proof of the following lemma can be found in [1].

Lemma 4.2. The numbers $\xi, \beta>1$ can be chosen a priori only in terms of $m, N$ so that

$$
\forall k, \rho_{0}>0, \quad \varphi_{t}-\Delta \varphi^{m} \leq 0, \quad \text { a.e. } \mathbf{R}^{N} \times\left(0, \frac{k^{1-m} \rho_{0}^{2}}{m \xi}\right) .
$$

Remark 4.1. The constants $\xi, \beta$ are "stable" as $m \rightarrow 1$.

4-(III). Subsolutions of the $p$-Laplacian equation. We construct here analogues of the functions $\psi$ and $\varphi$. Let $b, k, \mu, \theta>0$ and consider the cylindrical domain with annular cross section

$$
Q(\theta) \equiv\left\{\frac{\mu}{b^{p-1}} k^{p-2}<|x|^{p}<1\right\} \times\{0, \theta\}
$$

where $\mu, b, k$ are so that $\mu k^{p-2} / b^{p-1}<1$.

Consider the function

$$
\Psi \equiv \frac{k\left(1-|x|^{2}\right)_{+}^{p /(p-1)}}{\left[1+k^{(2-p) /(p-1)} b\left(|x|^{p} / t\right)^{1 /(p-1)}\right]^{(p-1) /(2-p)}} .
$$

Lemma 4.3. The constant $b=b(N, p)$ can be chosen a priori only dependent upon $N$ and $p$, so that

$$
\forall k>0, \forall \mu>0 \text { satisfying (4.22), }
$$

$$
\Psi_{t}-\operatorname{div}\left(|D \Psi|^{p-2} D \Psi\right) \leq 0 \quad \text { a.e. } Q(\theta), \quad \theta=\min \left\{\left(\frac{\kappa}{2 p}\right)^{p-1} \mu ; k^{2-p}\right\}
$$

The proof is similar to that of Lemma 4.1 and we postpone it to Appendix B.

The analog of the function $\varphi$ in (4.19), for equation (1.2) is

$$
\begin{aligned}
\Phi(x, t) & =\frac{k \rho_{0}^{p \xi /(p-1)}}{R(t)^{\xi /(p-1)}}\left[1-\left(\frac{|x|^{p}}{R(t)}\right)^{1 /(p-1)}\right]_{+}^{\beta /(p-1)}, \\
R(t) & =\left(\frac{b t}{k^{2-p}}+\rho_{0}^{p}\right),
\end{aligned}
$$

where $k, b, \xi, \beta, \rho_{0}$ are positive numbers.

Lemma 4.4. The numbers $b, \xi, \beta>1$ can be chosen a priori only in terms of $p, N$, so that

(4.25) $\forall k, \rho_{0}>0, \quad \Phi_{t}-\operatorname{div}\left(|D \Phi|^{p-2} D \Phi\right) \leq 0, \quad$ a.e. $\mathbf{R}^{N} \times\left(0, \frac{k^{2-p} \rho_{0}^{p}}{b \xi}\right)$.

The proof is analogous to that of Lemma 3.1 of [1].

Remark 4.2. Also $\xi, \beta$ are "stable" as $p \rightarrow 2$. 


\section{SPACE-Time configurations}

Locally bounded weak solutions of (1.1) are locally Hölder continuous in the interior of their domain of definition, $\forall 0<m<1$. When $m>(N-2)_{+} / N$, i.e., $\kappa>0$, this result exploits a space-time geometry whose precise configuration is needed in what follows.

Let $(\bar{x}, \bar{t}) \in \Omega_{T}, R_{0}>0, B_{R_{0}} \equiv\left\{|x-\bar{x}|<R_{0}\right\}$ and consider the cylinder $Q_{R_{0}} \equiv B_{R_{0}} \times\left\{\bar{t}-\omega_{0}^{1-m} R_{0}^{2}, \bar{t}\right\}$, where $\omega_{0}>0$ is any number satisfying

$$
\underset{Q_{R_{0}}}{\operatorname{ess} \sup } u \leq \omega_{0} .
$$

For each $(\bar{x}, \bar{t}) \in \Omega_{T}, \forall R_{0}>0$ such a cylinder can be constructed (see [6]) provided $R_{0}$ is so small that $Q_{R_{0}} \subset \Omega_{T}$.

Proposition 5.1. There exist numbers $\eta_{0} \in(0,1), C_{0}$ depending only upon $N, m$ satisfying the following.

For given $R_{0}, \omega_{0}$ construct the sequences

$$
R_{n}=C_{0}^{-n} R_{0}, \quad \omega_{n}=\eta_{0} \omega_{n-1}, \quad n=1,2, \ldots,
$$

and the family of nested cylinders

$$
Q_{n} \equiv B_{R_{n}} \times\left\{\bar{t}-\omega_{n}^{1-m} R_{n}^{2}, \bar{t}\right\} .
$$

Then

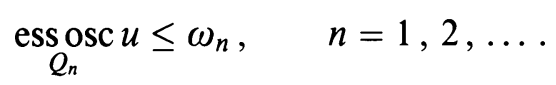

The proposition will be proved in Appendix B.

The construction in (5.1)-(5.3) implies the Hölder continuity of $u$ via an interpolation argument (see $[6,16])$. In particular we have

$$
\begin{gathered}
\exists \text { a constant } A=A(N, m) \text { such that } \forall 0<\rho<R_{0}, \\
\quad \underset{B_{\rho}}{\operatorname{essosc} u(\cdot, \bar{t}) \leq A \omega_{0}\left(\frac{\rho}{R_{0}}\right)^{\alpha},}
\end{gathered}
$$

where $\alpha=-\log _{C_{0}} \eta_{0}$.

Remark 5.1. The constant $A$ can be quantitatively determined a priori only in terms of $C_{0}, \eta_{0}$.

Remark 5.2. The proof in Appendix B shows that

$$
\begin{aligned}
& C_{0}(N, m) \rightarrow \infty \quad \text { as } m \rightarrow \frac{(N-2)_{+}}{N}, \\
& \eta_{0}(N, m) \rightarrow 1 \quad \text { as } m \rightarrow \frac{(N-2)_{+}}{N},
\end{aligned}
$$

i.e., as $\kappa=(N(m-1)+2) \rightarrow 0$. However, such constants are "stable" as $m \rightarrow 1$, i.e., $C_{0}(N, m) \rightarrow C_{0}(N, 1)<\infty$ and $\eta_{0}(N, m) \rightarrow \eta_{0}(N, 1) \in(0,1)$ (see also $\S 1$-(iii) of [6]).

Even though local solutions $u$ of (1.1) with no sign restriction and for all $m \in$ $(0,1)$ are in $C_{\mathrm{loc}}^{\alpha}\left(\Omega_{T}\right)$ for some $\alpha \in(0,1)$, the specific form of Proposition 
5.1 holds only for nonnegative solutions and for $m>(N-2)_{+} / N$, i.e., $\kappa>0$ (see Appendix B for further details).

\section{Proof of the Harnack inequality}

We let $u$ be a nonnegative locally bounded weak solution of (1.1) in $\Omega_{T}$. Let $P_{0} \equiv\left(x_{0}, t_{0}\right) \in \Omega_{T}$, assume that $u_{0} \equiv u\left(P_{0}\right)>0$ and construct the cylinder

$$
Q_{R}\left(u\left(P_{0}\right)\right) \equiv\left\{\left|x-x_{0}\right|<R\right\} \times\left\{t_{0}-\left[u\left(P_{0}\right)\right]^{1-m} R^{2}, t_{0}+\left[u\left(P_{0}\right)\right]^{1-m} R^{2}\right\} .
$$

We assume $R$ is so small that $Q_{4 R}\left(u\left(P_{0}\right)\right) \subset \Omega_{T}$. Without loss of generality we may assume that $\left(x_{0}, t_{0}\right) \equiv(0,0)$. The change of variable

$$
\xi=\frac{x-x_{0}}{R}, \quad \tau=\frac{t-t_{0}}{\left[u\left(P_{0}\right)\right]^{1-m} R^{2}}, \quad v=\frac{u}{\left[u\left(P_{0}\right)\right]},
$$

maps $Q_{4 R}\left(u\left(P_{0}\right)\right)$ into $Q_{4} \equiv B_{4} \times(-4,4)$, and the transformed function $v$ satisfies

$$
\left\{\begin{array}{l}
v_{\tau}-\Delta_{\xi} v^{m}=0 \quad \text { in } Q_{4} \\
v(0,0)=1
\end{array}\right.
$$

Denoting again with $x, t, u$ the transformed coordinates and the new function, to prove the theorem it will suffice to show that we can determine a priori constants $\gamma_{0}, \delta \in(0,1)$ depending only upon $N$ and $m$ such that

$$
\inf _{x \in B_{1}} u(x, \delta) \geq \gamma_{0} \text {. }
$$

6-(I). Locating the sup of $u$ in $Q_{4}$. For $s \in(0,1)$ construct the family of "increasing" cylinders

$$
Q_{s} \equiv\{|x|<s\} \times\{-\delta s, 0\},
$$

and the numbers

$$
M_{s} \equiv\|u\|_{\infty, Q_{s}}, \quad N_{s} \equiv(1-s)^{-2 /(1-m)} .
$$

Remark 6.1. Since $u \in L_{\mathrm{loc}}^{\infty}\left(\Omega_{T}\right), M_{0}=N_{0}$ and $N_{s} \rightarrow \infty$ as $s \rightarrow 1$, the equation $M_{s}=N_{s}$ has a largest root, say $s_{0}$. Thus

$$
M_{s_{0}}=\left(1-s_{0}\right)^{-2 /(1-m)}, \quad M_{\left(1+s_{0}\right) / 2} \leq 2^{2 /(1-m)}\left(1-s_{0}\right)^{-2 /(1-m)} .
$$

Since $u \in C_{\mathrm{loc}}^{\alpha}\left(\Omega_{T}\right), M_{s_{0}}$ is achieved at some point $(\bar{x}, \bar{t}) \in \bar{Q}_{s_{0}}$ and in view of $(6.2)$

$$
\sup _{|x-\bar{x}| \leq\left(1-s_{0}\right) / 2} u(x, \bar{t}) \leq 2^{2 /(1-m)}\left(1-s_{0}\right)^{-2 /(1-m)} .
$$

See Figure 2.

Remark 6.2. Here $\delta \in(0,1)$ is a small number to be chosen and has the effect of rendering "flat" the boxes $Q_{s}$; i.e., points in $Q_{s}, s \in(0,1)$ are at most $\delta s$ away from the hyperplane $t=0$.

Lemma 6.1. There exist a small positive number $\varepsilon \in(0,1)$ that can be determined a priori only in terms of $N$ and $m$, such that

$$
u(x, \bar{t}) \geq \frac{1}{2}\left(1-s_{0}\right)^{-2 /(1-m)}, \quad \forall|x-\bar{x}|<\varepsilon\left(1-s_{0}\right) .
$$

Proof. Set $\left(1-s_{0}\right) / 2=4 R$ and construct the box

$$
Q_{4 R}^{*} \equiv\{|x-\bar{x}|<4 R\} \times\{\bar{t}-4, \bar{t}\} .
$$


$(0,0)$

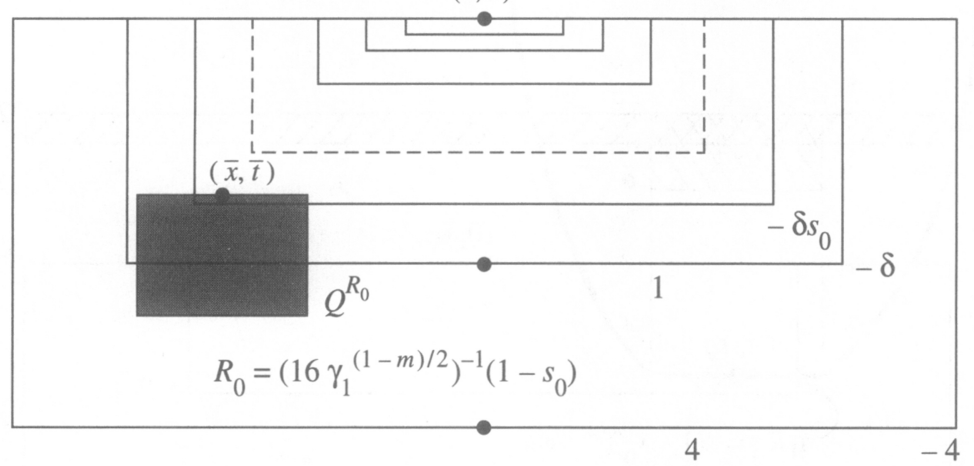

FIGURE 2

Apply to such a box the estimate (3.8) with the appropriate change of variables, to obtain

$$
\sup _{Q_{R}} u(x, t) \leq \gamma\left(\int_{B_{R}} u(x, \bar{t}) d x\right)^{2 / \kappa}+\gamma R^{-2 /(1-m)},
$$

where

$$
Q_{R} \equiv\{|x-\bar{x}|<R\} \times\{\bar{t}-1, \bar{t}\} .
$$

In view of (6.3) and the definition of $R$

$$
\sup _{Q_{\left(1-s_{0}\right) / 8}} u(x, t) \leq \gamma_{1}\left(1-s_{0}\right)^{-2 /(1-m)},
$$

where $\gamma_{1}=\gamma_{1}(m, N)$.

Next consider the cylinder

$$
\left\{\begin{array}{l}
Q_{R_{0}} \equiv\left\{|x-\bar{x}|<R_{0}\right\} \times\left\{\bar{t}-\gamma_{1}^{1-m}\left(1-s_{0}\right)^{-2} R_{0}^{2}, \bar{t}\right\}, \\
R_{0}=\left(16 \gamma_{1}^{(1-m) / 2}\right)^{-1}\left(1-s_{0}\right) .
\end{array}\right.
$$

By virtue of such a construction we have

$$
\|u\|_{\infty}, Q_{R_{0}} \leq \gamma_{1}\left(1-s_{0}\right)^{-2 /(1-m)},
$$

and $Q_{R_{0}}$ satisfies the space-time configuration of $\S 5$. Therefore by (5.4) we conclude that

$$
\begin{aligned}
& \forall 0<\rho<R_{0} \quad \forall|x-\bar{x}|<\rho, \quad \text { at the level } \bar{t} \\
& u(x, \bar{t}) \geq u(\bar{x}, \bar{t})-A \gamma_{1}\left(1-s_{0}\right)^{-2 /(1-m)}\left(\frac{\rho}{R_{0}}\right)^{\alpha} .
\end{aligned}
$$

Since $u(\bar{x}, \bar{t})=\left(1-s_{0}\right)^{-2 /(1-m)}$, by taking $\rho=\eta R_{0}, \eta \in(0,1)$ we find

$$
\begin{aligned}
u(x, \bar{t}) & \geq\left(1-s_{0}\right)^{-2 /(1-m)}\left(1-A \gamma_{1} \eta^{\alpha}\right), \\
\forall|x-\bar{x}| & \leq \eta R_{0} \equiv \eta\left(16 \gamma_{1}^{(1-m) / 2}\right)^{-1}\left(1-s_{0}\right),
\end{aligned}
$$

and the lemma follows by taking $\eta$ small enough.

6-(II). Time-expansion of positivity. The previous arguments are independent of the number $\delta$ (see Remark 6.2). We will now determine $\delta$. 


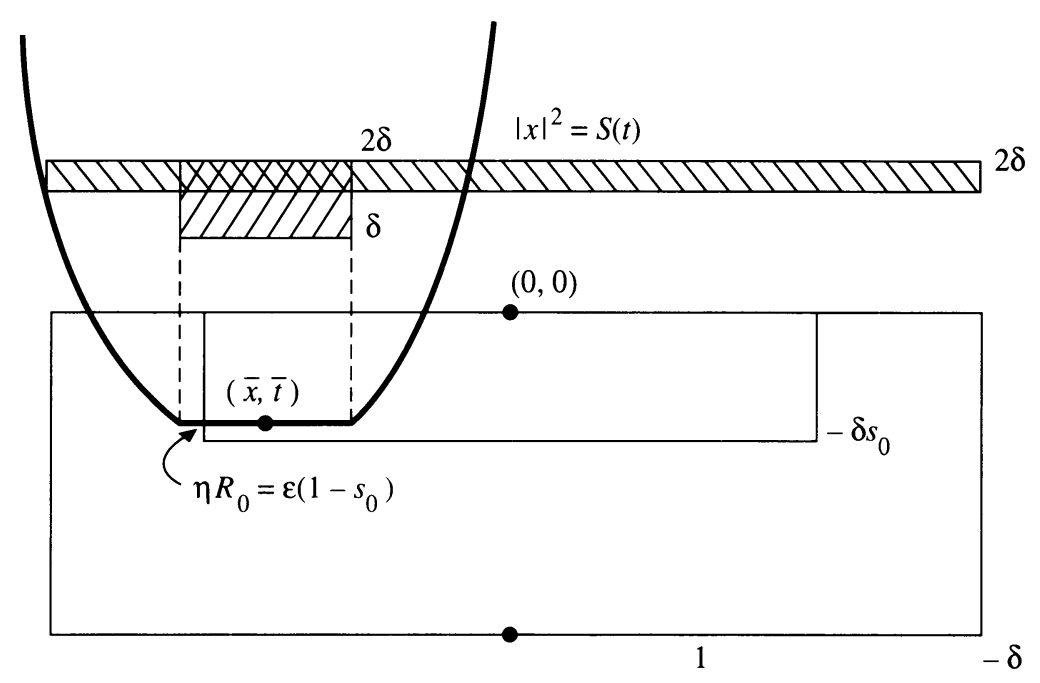

FigURE 3

Lemma 6.2. There exist small positive numbers $c_{0}, \delta$ that can be determined a priori only in terms of $N, m$, such that

$$
\begin{aligned}
& u(x, t) \geq c_{0}\left(1-s_{0}\right)^{-2 /(1-m)}, \\
& \forall|x-\bar{x}|<\varepsilon\left(1-s_{0}\right), \quad \forall \delta \leq t \leq 2 \delta .
\end{aligned}
$$

Proof. After a translation assume $(\bar{x}, \bar{t}) \equiv(0,0)$ and consider the comparison function $\varphi$ defined in (4.9) with the choices

$$
k=\frac{1}{2}\left(1-s_{0}\right)^{-2 /(1-m)}, \quad \rho_{0}=\varepsilon\left(1-s_{0}\right) .
$$

The function $\varphi$ is a subsolution of $(1.1)$ in $\mathbf{R}^{N} \times(0,3 \delta)$ where

$$
3 \delta=\frac{k^{1-m} \rho_{0}^{2}}{m \xi}=\frac{\varepsilon^{2} 2^{-(1-m)}}{m \xi} .
$$

For $t=0$ by virtue of Lemma 6.1, $u \geq \varphi$ and $\varphi$ vanishes on $|x|^{2}=S(t)$, $t>0$. Therefore by the comparison principle

$$
u \geq \varphi \text { in }\left\{|x|^{2}<S(t)\right\} \times\{0<t<3 \delta\} .
$$

In particular for $\delta<t<3 \delta$ and $|x| \leq \varepsilon\left(1-s_{0}\right)$,

$$
\begin{aligned}
u(x, t) & \geq \frac{\frac{1}{2}\left(1-s_{0}\right)^{-2 /(1-m)}}{\left[3 m \delta 2^{(1-m)}+1\right]^{\xi / m}}\left(1-\frac{1}{\left(m \delta 2^{1-m}+1\right)}\right)^{\beta / m} \\
& \equiv c_{0}\left(1-s_{0}\right)^{-2 /(1-m)} .
\end{aligned}
$$

In terms of the original coordinates

$$
\begin{aligned}
u(x, t) & \geq c_{0}\left(1-s_{0}\right)^{-2 /(1-m)}, \quad|x-\bar{x}|<\varepsilon\left(1-s_{0}\right), \\
\forall \bar{t}+\delta & <t<\bar{t}+3 \delta .
\end{aligned}
$$

The location of $\bar{t}$ in the box $Q_{s_{0}}$ is only known qualitatively. However, as $\bar{t}$ ranges over $(-\delta, 0)$, the intervals $[\bar{t}+\delta<t<\bar{t}+3 \delta]$ have the common intersection $[\delta<t<2 \delta]$ and the lemma is proved. (See Figure 3.) 
6-(III). The final step: sidewise expansion of positivity. Let $Q^{*}$ be the box (see Figure 1)

$$
Q^{*}(\bar{x}, \delta) \equiv\left\{|x-\bar{x}|<\varepsilon\left(1-s_{0}\right)\right\} \times\{\delta, 2 \delta\} .
$$

We will expand the positivity set of $u$ over the ball $\{|x|<1\}$ at the time level $t=2 \delta$. For this it will suffice to prove that there exist a constant $\gamma_{0}=\gamma_{0}(N, m)$ depending only upon $N$ and $m$ such that

$$
u(x, 2 \delta) \geq \gamma_{0}, \quad \forall|x-\bar{x}|<2 .
$$

After a translation we may assume that the centre-bottom of $Q^{*}(\bar{x}, \delta)$ is $(0,0)$. Consider the comparison function $\psi$ given in equation (4.3) with $k=$ $c_{0}\left(1-s_{0}\right)^{-2 /(1-m)}$, where $c_{0}$ is determined in Lemma 6.2.

Observe first that if $\psi$ is a subsolution of $(1.1)$ in $Q(\theta)$, via the change of variable $x \rightarrow \lambda x, t \rightarrow \lambda^{2} t$, the functions

$$
\psi_{\lambda} \equiv \frac{\lambda^{-2 \alpha / m} k\left(\lambda^{\alpha}-|x|^{\alpha}\right)_{+}^{2 / m}}{\left(1+k^{1-m} b|x|^{2} / t\right)^{1 /(1-m)}}
$$

are subsolutions of (1.1) in the annular regions

$$
Q^{(\lambda)} \equiv\left\{\frac{\mu}{b} k^{m-1} \lambda^{2}<|x|^{2}<\lambda^{2}\right\} \times\left\{0, \lambda^{2} \theta\right\}
$$

for the same values of $b, \mu, \theta$ as determined in Lemma 4.1.

The number $\mu$ here can be determined by imposing

$$
\frac{\mu}{b} k^{m-1} \leq \frac{\varepsilon^{2}\left(1-s_{0}\right)^{2}}{\lambda^{2}}, \quad \text { i.e., } \quad \mu \leq \frac{b}{c_{0}^{1-m}} \frac{\varepsilon^{2}}{\lambda^{2}} .
$$

We choose

$$
\mu=\min \left\{\frac{1}{4} ; \frac{b}{c_{0}^{1-m}} \frac{\varepsilon^{2}}{\lambda^{2}}\right\}
$$

and pick $\theta$ according to (4.12). By further restricting either $\mu$ or the number $\delta$ of Lemma 6.2 we may assume that $\theta=\lambda^{2} \delta$. Take $\lambda=3$ in (6.11). Then on the parabolic boundary of $Q^{(3)}$ we have

(i) $|x|=3,0<t \leq \theta, u(x, t) \geq \psi_{3}(x, t)=0$,

(ii) $|x|=\varepsilon\left(1-s_{0}\right), 0<t \leq \theta$ by Lemma 6.2 ,

$$
\psi_{3}(\ddot{i}, t) \leq c_{0}\left(1-s_{0}\right)^{-2 /(1-m)} \leq u(x, t),
$$

(iii) for $\varepsilon\left(1-s_{0}\right)<|x|<3, \psi_{3}(x, 0)=0$.

Therefore by the comparison principle for $t=\delta, \forall|x|<2$

$$
u(x, \delta) \geq \frac{c_{0}\left(1-s_{0}\right)^{-2 /(1-m)}\left(3^{\alpha}-2^{\alpha}\right)^{2 / m} 3^{-2 \alpha / m}}{\left(1+\left[c_{0}\left(1-s_{0}\right)^{-2 /(1-m)}\right]^{1-m} 4 b / \delta\right)^{1 /(1-m)}} \equiv \gamma_{0}(N, m)
$$

6-(IV). The case of $m$ near 1 . The previous proof makes use of (3.8) so that the constants $\delta, \gamma_{0}$ in (6.1) tend to zero as $m \rightarrow 1$ (see Remark 3.5). We will give a proof of (6.1) that holds only for $m$ near 1 but it is "stable" for $m \rightarrow 1$, i.e., $\delta(N, m), \gamma_{0}(N, m) \rightarrow \delta(N, 1), \gamma_{0}(N, 1) \in(0,1)$ as $m \rightarrow 1$. 
Theorem 6.1. There exists $m_{*} \in\left((N-2)_{+} / N, 1\right)$ and constants $\gamma_{0}^{*}=\gamma_{0}^{*}\left(N, m_{*}\right)$, $\delta_{*}=\delta_{*}\left(N, m_{*}\right) \in(0,1)$ such that if $m_{*} \leq m<1$

$$
u\left(x, \delta_{*}\right) \geq \gamma_{0}^{*}, \quad \forall x \in B_{1} .
$$

Proof. As before construct the family of increasing cylinders $Q_{s} \equiv\{|x|<s\} \times$ $\{-\delta s, 0\}$ and the numbers

$$
M_{s} \equiv\|u\|_{\infty, Q_{s}}, \quad N_{s} \equiv(1-s)^{-\gamma},
$$

where $\gamma$ is a positive number to be chosen. The definition of the numbers $N_{s}$ differs from that in $\S 6-(\mathrm{I})$ in the arbitrariety of the exponent $\gamma$.

Since $u \in L_{\text {loc }}^{\infty}\left(\Omega_{T}\right), M_{0}=N_{0}$, and $N_{s} \rightarrow \infty$ as $s \rightarrow 1$, the equation $M_{s}=N_{s}$ has a largest root, say $s_{0}$. Thus

$$
M_{s_{0}}=\left(1-s_{0}\right)^{-\gamma}, \quad M_{\left(1+s_{0}\right) / 2} \leq 2^{\gamma}\left(1-s_{0}\right)^{-\gamma} .
$$

Since $u \in C_{\mathrm{loc}}^{\alpha}\left(\Omega_{T}\right), M_{s_{0}}$ is achieved at some point $(\bar{x}, \bar{t}) \in \bar{Q}_{s_{0}}$ and in view of $(6.13)$

$$
u(x, t) \leq 2^{\gamma}\left(1-s_{0}\right)^{-\gamma}, \quad|x-\bar{x}|<\frac{1-s_{0}}{2}, \quad \bar{t}-\delta \frac{1-s_{0}}{2}<t<\bar{t} .
$$

Let

$$
R_{0}=\frac{1}{2} 2^{-\gamma(1-m) / 2}\left(1-s_{0}\right)^{\gamma(1-m) / 2}\left(1-s_{0}\right),
$$

and consider the box

$$
Q_{0} \equiv\left\{|x-\bar{x}|<R_{0}\right\} \times\left\{\bar{t}-\left[2^{\gamma}\left(1-s_{0}\right)^{-\gamma}\right]^{(1-m) / 2} R_{0}^{2}, \bar{t}\right\} .
$$

Assume first $s_{0}$ is so close to 1 that $\delta \geq \frac{1}{2}\left(1-s_{0}\right)$, so that from the definitions of $Q_{s}, R_{0}$ we have $Q_{0} \subset Q_{\left(1+s_{0}\right) / 2}$.

By virute of (6.14) $\|u\|_{\infty}, Q_{0} \leq 2^{\gamma}\left(1-s_{0}\right)^{-\gamma}$ and $Q_{0}$ satisfies the space-time configuration of Proposition 5.1. Therefore

$$
\forall|x-\bar{x}|<\rho, \quad|u(x, \bar{t})-u(\bar{x}, \bar{t})|<A 2^{\gamma}\left(1-s_{0}\right)^{-\gamma}\left(\frac{\rho}{R_{0}}\right)^{\alpha} .
$$

By taking $\rho=\eta R_{0}$ and then $\eta$ sufficiently small we have

Lemma 6.1'. There exists a small positive number $\varepsilon \in(0,1)$ that can be determined a priori only in terms of $N, m$ such that

$$
u(x, \bar{t}) \geq \frac{1}{2}\left(1-s_{0}\right)^{-\gamma}, \quad \forall|x-\bar{x}|<\varepsilon\left(1-s_{0}\right)^{(\gamma(1-m)+2) / 2} .
$$

Remark 6.3. The constant $\varepsilon$ depends upon $\gamma$ but it is "stable" as $m \rightarrow 1$ since no use has been made of (3.8).

We now expand the positivity set of $u$ by using the comparison function $\varphi$ in (4.9) with the choices

$$
k=\frac{1}{2}\left(1-s_{0}\right)^{-\gamma}, \quad \rho_{0}=\varepsilon\left(1-s_{0}\right)^{(\gamma(1-m)+2) / 2} .
$$

Proceeding as before we find

$$
\begin{aligned}
& u\left(x, t_{1}\right) \geq \tau\left(1-s_{0}\right)^{-\gamma}, \\
&|x-\bar{x}|<(1+\sigma) \rho_{0}=\varepsilon(1+\sigma)\left(1-s_{0}\right)^{(\gamma(1-m)+2) / 2} . \\
& t_{1}=\bar{t}+\frac{\varepsilon^{2}}{2^{(1-m)} m \xi}\left(1-s_{0}\right)^{\gamma(1-m)+2}\left(1-s_{0}\right)^{-\gamma(1-m)}=\bar{t}+\frac{\varepsilon^{2}\left(1-s_{0}\right)^{2}}{2^{1-m} m \xi},
\end{aligned}
$$


where $\sigma, \tau$ are numbers that can be determined a priori only in terms of $N, m$ and are "stable" as $m \rightarrow 1$.

We apply again the comparison function $\varphi$ starting from the level $t_{1}$, with $\rho_{0}$ replaced by $(1+\sigma) \rho_{0}$ and $k$ replaced by $\tau\left(1-s_{0}\right)^{-\gamma}$, to obtain

$$
\begin{aligned}
& u\left(x, t_{2}\right) \geq \tau^{2}\left(1-s_{0}\right)^{-\gamma}, \\
& \forall|x-\bar{x}|<\left(1+\sigma^{2}\right) \rho_{0}=\varepsilon(1+\sigma)^{2}\left(1-s_{0}\right)^{(\gamma(1-m)+2) / 2} . \\
& t_{2}=t_{1}+\frac{\varepsilon^{2}\left(1-s_{0}\right)^{2}(1+\sigma)^{2} \tau^{1-m}}{2^{(1-m)} m \xi} .
\end{aligned}
$$

By iteration we find a sequence of expanding balls

$$
B_{n} \equiv\left\{|x-\bar{x}|<\varepsilon(1+\sigma)^{n}\left(1-s_{0}\right)^{(\gamma(1-m)+2) / 2}\right\}, \quad n=1,2, \ldots,
$$

and time levels

$$
t_{0}=\bar{t}, \quad t_{n+1}=t_{n}+\frac{\varepsilon^{2}\left(1-s_{0}\right)^{2}}{2^{(1-m)} m \xi}(1+\sigma)^{2 n} \tau^{(1-m) n},
$$

satisfying

$$
u\left(x, t_{n}\right) \geq \tau^{n}\left(1-s_{0}\right)^{-\gamma}, \quad \text { in } B_{n} .
$$

We choose $n$ so large that $B_{n}$ covers the ball centered at zero of radius 1 , i.e.,

$$
\varepsilon(1+\sigma)^{n}\left(1-s_{0}\right)^{(\gamma(1-m)+2) / 2}=2,
$$

where we have assumed, without loss of generality that the solution of $(6.20)$ is an integer $n$. Then from $\left(6.17_{n}\right)$ for $|x|<1$

$$
u\left(x, t_{n}\right) \geq \tau^{n}\left(1-s_{0}\right)^{-\gamma} \equiv\left(\frac{\varepsilon}{2}\right)^{2 \gamma /(\gamma(1-m)+2)}\left[\tau(1+\sigma)^{2 \gamma /(\gamma(1-m)+2)}\right]^{n} .
$$

Choose $\gamma$ so large to satisfy $\tau(1+\sigma)^{2 \gamma / 3}=1$. Then if $m$ is so close to 1 as to satisfy $\gamma(1-m)<1$, we obtain from $(6.21)$

$$
u\left(x, t_{n}\right) \geq \gamma_{0}^{*} \equiv\left(\frac{\varepsilon}{2}\right)^{2 \gamma /(\gamma(1-m)+2)} .
$$

It remains to estimate the time level $t_{n}$. From (6.19)

$$
t_{n}=\bar{t}+\frac{\varepsilon^{2}\left(1-s_{0}\right)^{2}}{2^{(1-m)} m \xi} \sum_{i=1}^{n}\left((1+\sigma)^{2} \tau^{(1-m)}\right)^{(i-1)} .
$$

Then if $m$ is so close to 1 as to satisfy in addition $(1+\sigma)^{2} \tau^{(1-m)}>1$ we find from (6.22) and (6.19), (6.20)

$$
\begin{aligned}
t_{n} & =\bar{t}+\frac{\varepsilon^{2}\left(1-s_{0}\right)^{2}}{2^{(1-m)} m \xi} \frac{\left[(1+\sigma)^{2} \tau^{(1-m)}\right]^{n-1}}{(1+\sigma)^{2} \tau^{(1-m)}-1} \\
& \geq-\delta+\frac{4}{2^{(1-m)} m \xi}\left[\tau^{n}\left(1-s_{0}\right)^{-\gamma}\right]^{1-m} \\
& \geq-\delta+\frac{4}{2^{(1-m)} m \xi}\left(\frac{\varepsilon}{2}\right)^{2 \gamma /(\gamma(1-m)+2)}
\end{aligned}
$$


To prove the theorem we choose $\delta$ so small that

$$
-\delta+\frac{4}{2^{(1-m)} m \xi}\left(\frac{\varepsilon}{2}\right)^{2 \gamma /(\gamma(1-m)+2)}=\frac{\delta}{2} \equiv \delta_{*} .
$$

Finally, the numbers $\delta, \gamma$ being fixed, if $\delta<\left(1-s_{0}\right)$ we have $\left(1-s_{0}\right)^{-\gamma} \leq$ $(2 \delta)^{-\gamma}$. Therefore we have a quantitative estimate of the sup of $u$ in the cylinder $Q_{0}$. We may assume without loss of generality that $\frac{1}{2} \leq s_{0}<1$. Indeed if not, from the definition of the numbers $M_{s}$ and $N_{s}$ it would follow that $\|u\|_{\infty, Q_{1 / 2}} \leq 2^{\gamma}$.

In either case we conclude that there exists an absolute constant $V$ such that

$$
\|u\|_{\infty, Q_{1 / 2}} \leq V, \quad u(0,0)=1 .
$$

By the Hölder continuity

$$
u(x, 0)>c_{0}, \quad|x|<\varepsilon_{0},
$$

where the numbers $c_{0}, \varepsilon_{0}$ depend upon $V$ and can be a priori determined by applying Proposition 5.1. We are now in a position of expanding the positivity set of $u$ by using the comparison function $\varphi$ of (4.9).

Remark 6.4. Our results generalize to the case when the right-hand side of (1.1), (1.2) is replaced by $f(x, t, u)$ provided $0 \leq f(x, t, u) \leq F(1+|u|)$ for a constant $F$. The growth conditions insures the Hölder continuity as well as the sup estimates of $\S 3$. The positivity permits the application of the comparison principle.

\section{APPENDIX A: ON THE HÖLDER CONTINUITY OF SOLUTIONS}

Let $u$ be a locally bounded local weak solution of $(1.1)$ in $\Omega_{T}$, with no sign restriction and for any $m \in(0,1)$. Fix $h \in(0,1),(\bar{x}, \bar{t}) \in \Omega_{T}$ and construct the cylinder

$$
Q_{R_{0}}^{(h)} \equiv\left\{|x-\bar{x}|<R_{0}\right\} \times\left\{\bar{t}-\omega_{0}^{1-m} R_{0}^{2}, \bar{t}+h \omega_{0}^{1-m} R_{0}^{2}\right\},
$$

where $\omega_{0}$ is any positive number satisfying essosc $u_{Q_{R_{0}}^{(h)}} \leq \omega_{0}$, and $R_{0}$ is so small that $Q_{R_{0}}^{(h)} \subset \Omega_{T}$.

The Hölder continuity of $u$ can be established by adapting the arguments of [6, see also Remark (d), p. 323], leading to

Proposition A. There exist numbers $\eta_{0}, C_{0}$ depending only upon $N, m$, and $h$ satisfying:

for given $R_{0}, \omega_{0}$ construct the sequences

$$
\left\{\begin{array}{l}
R_{n}=C_{1}^{-n} R_{0} ; \quad \omega_{n}=\eta_{0} \omega_{n-1}, \quad n=1,2, \ldots, \\
\text { and the family of nested cylinders } \\
Q_{n}^{(h)} \equiv B_{R_{n}} \times\left\{\bar{t}-\omega_{n}^{1-m} R_{n}^{2}, \bar{t}+h \omega_{n}^{1-m} R_{n}^{2}\right\}
\end{array}\right.
$$

Then

$$
\underset{Q_{n}^{(h)}}{\operatorname{ess} \text { osc }} u \leq \omega_{n}, \quad n=1,2, \ldots
$$

The numbers $C_{0} \nearrow \infty$ and $\eta_{0} \nearrow 1$ as $h \rightarrow 0$. 
The new information contained in Proposition 5.1 is that if $u \geq 0$ and if $m>(N-2)_{+} / N$, then $h$ can be taken to be zero. This fact is connected to Remark 2.2 of $\S 2$. Indeed, if Proposition A were to hold with $h=0$ and $C_{0}<\infty, \eta_{0} \in(0,1)$, then a Harnack estimate would hold for all $m \in(0,1)$. We will point out, in the course of the proof of Proposition 5.1, where such a circumstance occurs.

A-(I). Proof of Proposition 5.1. The proof follows mainly the ideas of [6] and therefore familiarity with that work will be assumed. The new fact is an expansion of positivity (Lemma A.1, below) which is possible because of the comparison function $\psi$ of $\S 4$.

If $R, \nu>0$ consider the $N$-dimensional cube

$$
K_{R}(\bar{x}, \nu) \equiv\left\{\max _{1 \leq i \leq N}\left|x_{i}-\bar{x}_{i}\right|<\nu^{(1-m) / 2} R\right\},
$$

and the $(N+1)$-dimensional box

$$
C_{R}\left(\bar{x}, \bar{t} ; \nu ; \omega_{0}\right) \equiv K_{R}(\bar{x}, \nu) \times\left\{\bar{t}-\omega_{0}^{1-m} R^{2}, \bar{t}\right\},
$$

where $\omega_{0}$ is any number satisfying

$$
\underset{C_{8 R}\left(\bar{x}, \bar{t} ; \nu, \omega_{0}\right)}{\operatorname{ess} \text { osc }} u \leq \omega_{0} .
$$

Proposition 5.1 will be a consequence of

Proposition A.2. There exist $\nu>1, \eta_{0} \in(0,1)$ that can be determined a priori only in terms of $N$ and $m$ such that

$$
\underset{C_{R}\left(\bar{x}, \bar{t} ; \nu, \omega_{0}\right)}{\operatorname{ess} \text { osc }} u \leq \eta_{0} \omega_{0} \text {. }
$$

Proof. We let $\nu=2^{s^{*}}$, where $s^{*}$ is a large positive integer to be chosen only dependent on $m$ and $N$. Without loss of generality we may assume that

$$
\nu^{(1-m) / 2}=2^{s^{*}((1-m) / 2)}=\nu_{0}
$$

is an integer and, following [6], subdivide $K_{2 R}\left(\bar{x}, 2^{s^{*}}\right)$ into $\nu_{0}^{N}$ pairwise disjoint subcubes $K_{2 R}\left(x_{j}, 1\right), j=1,2, \ldots, \nu_{0}^{N}$. We consider also the boxes

$$
C_{2 R}^{(j)} \equiv K_{2 R}\left(x_{j}, 1\right) \times\left\{\bar{t}-\omega_{0}^{1-m}(2 R)^{2}, \bar{t}\right\}, \quad j=1,2, \ldots, \nu_{0}^{N} .
$$

The proof is based on the following two alternative statements.

Lemma A.1. There exist numbers $\alpha_{0}=\alpha_{0}(N, m)$ and $\eta_{0}=\eta_{0}(N, m) \in(0,1)$ such that,

$$
\begin{gathered}
\text { If for some } 1 \leq j \leq \nu_{0}^{N}, \\
\operatorname{meas}\left\{(x, t) \in C_{2 R}^{(j)} \mid u(x, t)>\omega_{0} / 2\right\} \leq \alpha_{0} \operatorname{meas}\left\{C_{2 R}^{(j)}\right\},
\end{gathered}
$$

then

$$
u(x, t) \geq \eta_{0} \omega, \quad(x, t) \in C_{R / 2}\left(\bar{x}, \bar{t} ; 2^{s^{*}}, \omega_{0}\right) .
$$

Lemma A.2. If (A.5) fails to hold for all $j=1,2, \ldots, \nu_{0}^{N}$, then

$$
u(x, t) \leq \operatorname{Coss}_{C_{R}\left(\bar{x}, \bar{t} ; 2^{5^{*}}, \omega_{0}\right)} u-\left(1-\eta_{0}\right) \omega_{0}, \forall(x, t) \in C_{R / 2}\left(\bar{x}, \bar{t} ; 2^{s^{*}}, \omega_{0}\right) .
$$


The proof of Lemma A. 2 follows step by step the same arguments of $\S 5$ of [6] and we omit it.

Proof of Lemma A.1. By adapting the proof of Lemma 3.1 of [6] we deduce

$$
u(x, t) \geq \frac{1}{4} \omega_{0}, \quad(x, t) \in C_{R}^{(j)} .
$$

We switch from the cubes $K_{R}\left(x_{j}, 1\right), K_{8 R}\left(\bar{x}, 2^{s^{*}}\right)$ to the balls

$$
B_{R}\left(x_{j}\right) \equiv\left\{\left|x-x_{j}\right|<R\right\}, \quad B_{8 R}\left(\bar{x}, 2^{s^{*}}\right) \equiv\left\{|x-\bar{x}|<2^{s^{*}((1-m) / 2)} 8 R\right\},
$$

and from $C_{R}^{(j)}$ and $C_{8 R}\left(\bar{x}, \bar{t} ; 2^{s^{*}} \omega_{0}\right)$ to the cylinders

$$
\begin{aligned}
Q_{R}^{(j)} & \equiv B_{R}\left(x_{j}\right) \times\left\{\bar{t}-\omega_{0}^{1-m} R^{2}, \bar{t}\right\} \\
Q_{8 R}\left(\bar{x}, \bar{t} ; 2^{s^{*}}, \omega_{0}\right) & \equiv B_{8 R}\left(\bar{x}, 2^{s^{*}}\right) \times\left\{\bar{t}-\omega_{0}^{1-m}(8 R)^{2}, \bar{t}\right\} .
\end{aligned}
$$

Thus inside the cylinder $Q_{8 R}\left(\bar{x}, \bar{t} ; 2^{s^{*}}, \omega_{0}\right)$ there exists a cylinder $Q_{R}^{(j)}$ such that

$$
u(x, t) \geq \omega_{0} / 4, \quad(x, t) \in Q_{R}^{(j)} .
$$

Consider the comparison function $\psi_{\lambda}\left(x-x_{*} ; t-t_{*}\right)$ defined in (4.13) with the choices

$$
\left\{\begin{array}{l}
k=\frac{1}{4} \omega_{0}, \quad \lambda=2^{s^{*}((1-m) / 2)}(4 R), \\
\left(x_{*}, t_{*}\right) \text { coincides with the bottom center } \\
\text { of } Q_{R}^{(j)}, \text { i.e., }\left(x_{j}, \bar{t}-\omega_{0}^{1-m} R^{2}\right)
\end{array}\right.
$$

The function $\psi_{\lambda}$ is defined in the annular region

$$
Q_{*}^{(\lambda)} \equiv\left\{R^{2}<\left|x-x_{*}\right|^{2}<\lambda^{2}(4 R)^{2}\right\} \times\left\{t_{*}, t_{*}+\lambda^{2} \theta\right\} .
$$

The condition $\lambda^{2} \mu k^{m-1} / b=R^{2}$ implies

$$
\mu=\frac{b \omega_{0}^{1-m}}{4^{3-m} 2^{s^{*}(1-m)}},
$$

and by (4.4) if $s^{*}$ is sufficiently large we choose

$$
\lambda^{2} \theta=\omega_{0}^{1-m} R^{2} .
$$

By the comparison principle, in view of (A.8) we have for $\left|x-x_{*}\right|^{2} \leq$ $2 R 2^{s^{*}((1-m) / 2)}$ and $t_{*}+\omega_{0}^{1-m} R^{2} / 4<t<t_{*}+\omega_{0}^{1-m} R^{2}$,

$$
\begin{aligned}
& u(x, t) \geq\left(\frac{3}{4}\right)^{\alpha / m} \frac{\omega_{0}}{4}\left(1+\left(\frac{\omega_{0}}{4}\right)^{1-m} \frac{4 R^{2} 2^{s^{*}(1-m)}}{\omega_{0}^{1-m} R^{2} / 4}\right)^{-1 /(1-m)}=\eta_{0} \omega_{0}, \\
& \eta_{0}=\eta_{0}\left(N, m, s^{*}\right) .
\end{aligned}
$$

Remark. The comparison function $\psi_{\lambda}$ holds for $\kappa>0$ and $\eta_{0}$ above depends upon the constants $b, \alpha$ of Lemma 4.1. From (4.9) and (4.12) it follows that $\alpha \rightarrow 0$ and $b \rightarrow \infty$ as $\kappa \rightarrow 0$, so that $\eta_{0} \rightarrow 0$ as $\kappa \rightarrow 0$.

\section{APPENDIX B: PROOF OF LEMMA 4.3}

The function $x \rightarrow \Psi(x, t)$ is radial and decreasing, so that writing (1.2) in polar coordinates we have

$$
\begin{aligned}
\mathscr{L}(\Psi) & \equiv \Psi_{t}-\operatorname{div}\left(|D \Psi|^{p-2} D \Psi\right) \\
& =\Psi_{t}+\left(\frac{N-1}{\rho}\right)\left(-\Psi^{\prime}\right)^{p-1}-(p-1)\left(-\Psi^{\prime}\right)^{p-2} \Psi^{\prime \prime}
\end{aligned}
$$


where

$$
\rho=|x|, \quad \Psi^{\prime}=\frac{d}{d \rho} \Psi, \quad \Psi^{\prime \prime}=\frac{d^{2}}{d \rho^{2}} \Psi
$$

We write

$$
\begin{aligned}
& \left(-\Psi^{\prime}\right)^{2-p} \mathscr{L}(\Psi)=\left(-\Psi^{\prime}\right)^{2-p} \Psi_{t}+\mathscr{R}(\Psi), \\
& \mathscr{R}(\Psi) \equiv \frac{N-1}{\rho}\left(-\Psi^{\prime}\right)-(p-1) \Psi^{\prime \prime},
\end{aligned}
$$

and calculate $\mathscr{R}(\Psi)$ as follows.

First we set

$$
\begin{gathered}
|z|=k^{(2-p) /(p-1)} b\left(\frac{|x|^{p}}{t}\right)^{1 /(p-1)}, \quad \mathscr{F}=1+|z|, \\
w=\frac{k}{\mathscr{F}(p-1) /(2-p)}, \quad v=\left(1-|x|^{2}\right)^{p /(p-1)}, \quad \Psi=w v .
\end{gathered}
$$

Then by direct calculation

(B.3)

$$
\left\{\begin{array}{l}
w^{\prime}=-\frac{p}{2-p}\left(\frac{w}{\rho}\right) \frac{|z|}{\mathscr{F}} \\
w^{\prime \prime}=\left(\frac{p}{2-p}\right)^{2}\left(\frac{w}{\rho^{2}}\right) \frac{|z|^{2}}{\mathscr{F} 2}+\frac{p}{2-p}\left(\frac{w}{\rho^{2}}\right) \frac{|z|}{\mathscr{F}}-\frac{p^{2}}{(2-p)(p-1)}\left(\frac{w}{\rho^{2}}\right) \frac{|z|}{\mathscr{F} 2}, \\
v^{\prime}=-\frac{2 \rho p}{p-1}\left(1-\rho^{2}\right)^{1 /(p-1)}, \\
v^{\prime \prime}=\frac{p}{(p-1)^{2}} 4 \rho^{2}\left(1-\rho^{2}\right)^{(2-p) /(p-1)}-\frac{2 p}{p-1}\left(1-\rho^{2}\right)^{1 /(p-1)} \\
\geq-\frac{2 p}{p-1}\left(1-\rho^{2}\right)^{1 /(p-1)} .
\end{array}\right.
$$

We calculate the expressions $\Psi^{\prime}=w v^{\prime}+w^{\prime} v$ and $\Psi^{\prime \prime}=w^{\prime \prime} v+2 w^{\prime} v^{\prime}+w v^{\prime \prime}$ from (B.3) and combine them into $\mathscr{R}(\Psi)$ to obtain

$$
\begin{aligned}
\mathscr{R}(\Psi) \leq & \frac{p}{2-p}\left(1-\rho^{2}\right)^{p /(p-1)}\left(\frac{w}{\rho^{2}}\right) \frac{|z|}{\mathscr{F}} \\
& \times\left\{N-1-(p-1)\left[\frac{p}{2-p} \frac{|z|}{\mathscr{F}}+1\right]+p \frac{1}{\mathscr{F}}\right\} \\
& +2 p w\left(1-\rho^{2}\right)^{1 /(p-1)}\left\{\frac{(N-1)}{p-1}-\frac{2 p}{2-p} \frac{|z|}{\mathscr{F}}+1\right\} .
\end{aligned}
$$

Rewrite the first factor in braces on the right-hand side of (B.4) as:

$$
\{\cdots\}=\left(N-\frac{p}{2-p} \frac{|z|}{\mathscr{F}}\right) .
$$

We will impose on $|z|$ to be so large that

$$
N-\frac{p}{2-p} \frac{|z|}{\mathscr{F}}<0
$$

This is possible since $N(p-2)+p \equiv \kappa>0$. 
The second term in braces on the right-hand side of (B.4) is negative if we choose $|z|$ to satisfy (B.5). If $N=1,2$ this is a direct consequence of (B.5). If $N \geq 3$

$$
\begin{aligned}
(p-1) & \left\{\frac{N-1}{p-1}-\frac{2 p}{2-p} \frac{|z|}{\mathscr{F}}+1\right\} \\
= & \left(N-\frac{p}{2-p} \frac{|z|}{\mathscr{F}}\right)+\frac{p}{2-p}(3-2 p) \frac{|z|}{\mathscr{F}}+(p-2) .
\end{aligned}
$$

The first term is negative in view of (B.5) and the second is negative since $p>2 N /(N+1)>\frac{3}{2}$ if $N \geq 3$. We drop the last negative term on the righthand side of (B.4) and estimate

$$
\mathscr{R}(\Psi) \leq \frac{p}{2-p} v\left(\frac{w}{\rho^{2}}\right) \frac{|z|}{\mathscr{F}}\left[N-\frac{p}{2-p} \frac{|z|}{\mathscr{F}}\right] .
$$

We return to (B.2) and estimate above the term $\left(-\Psi^{\prime}\right)^{2-p} \Psi_{t}$. First using (B.3)

$$
-\Psi^{\prime} \leq \frac{w}{\rho}\left(\frac{2 p}{p-1}+\frac{p}{2-p}\right) \equiv \gamma\left(\frac{w}{\rho}\right) .
$$

Also

Therefore

$$
\Psi_{t}=\frac{1}{2-p} v w \frac{|z|}{\mathscr{F}} \frac{1}{t}
$$

$$
\left(-\Psi^{\prime}\right)^{2-p} \Psi_{t} \leq \frac{\bar{\gamma}}{2-p}\left(\frac{w}{\rho}\right)^{2-p} v w \frac{|z|}{\mathscr{F}} \frac{1}{t} .
$$

We combine this with (B.6) into (B.2) and set

$$
\mathscr{L}^{*}(\Psi)=\left(-\Psi^{\prime}\right)^{2-p}\left[\Psi_{t}-\operatorname{div}\left(|D \Psi|^{p-2} D \Psi\right)\right] \frac{(2-p) \mathscr{F} \rho^{2}}{v w|z|}
$$

to obtain

$$
\mathscr{L}^{*}(\Psi) \leq \gamma w^{2-p} \frac{\rho^{p}}{t}+p\left[N-\frac{p}{2-p} \frac{|z|}{\mathscr{F}}\right] .
$$

From the definition of $w$ and $|z|$

$$
w^{2-p} \frac{\rho^{p}}{t}=\left(\frac{|z|}{1+|z|}\right)^{p-1} b^{1-p} \leq \frac{1}{b^{p-1}}
$$

and

$$
\mathscr{L}^{*}(\Psi) \leq \frac{\gamma}{b^{p-1}}+\frac{p}{2-p}\left[-\kappa+\frac{p}{\mathscr{F}}\right] .
$$

We will choose $|z|$ so large that $p / \mathscr{F} \leq \kappa / 2$. From the construction of the cylinder $Q(\theta)$ in (4.21) we have

$$
|x|^{p /(p-1)} k^{(2-p) /(p-1)} b \geq \mu^{1 /(p-1)}
$$

and to prove the lemma it will suffice to choose $0<t<\theta=(\kappa / 2 p)^{p-1} \mu$.

APPENDIX C: PROOF OF LEMMA 3.3

Let $|x|=\rho$ so that

$$
g=\frac{\left(\varepsilon^{2}-\rho^{2}\right)_{+}^{a_{1}}}{\rho^{N}\left|\ln \rho^{2}\right|^{a_{2}}}
$$


and compute within the set $\rho^{2}<\varepsilon^{2}$,

$$
\begin{aligned}
D g & =-\frac{g}{\rho^{2}} F x, \quad F=\left\{N+\frac{2 a_{2}}{\ln \rho^{2}}+\frac{2 a_{1} \rho^{2}}{\varepsilon^{2}-\rho^{2}}\right\}, \\
D F \cdot x & =-\frac{4 a_{2}}{\left(\ln \rho^{2}\right)^{2}}+\frac{4 a_{1} \rho^{2}}{\varepsilon^{2}-\rho^{2}}+\frac{4 a_{1} \rho^{4}}{\left(\varepsilon^{2}-\rho^{2}\right)^{2}}, \\
D g^{m} & =-\frac{m g^{m}}{\rho^{2}} F x, \quad \Delta g^{m}=\frac{m g^{m}}{\rho^{2}} \mathscr{H},
\end{aligned}
$$

where

$$
\mathscr{H}=m F^{2}-(N-2) F+\frac{4 a_{2}}{\left(\ln \rho^{2}\right)^{2}}-\frac{4 a_{1} \rho^{2}}{\varepsilon^{2}-\rho^{2}}-\frac{4 a_{1} \rho^{4}}{\left(\varepsilon^{2}-\rho^{2}\right)^{2}} .
$$

We estimate $\mathscr{H}$ below by using the fact that

$$
m=\frac{N-2}{N}, \quad N \geq 3 .
$$

We have $\forall \rho^{2}<\varepsilon^{2}$

$$
\begin{aligned}
\mathscr{H} \geq & \frac{(N-2)}{N} F^{2}-(N-2) F-\frac{4 a_{1} \rho^{2}}{\left(\varepsilon^{2}-\rho^{2}\right)_{+}}-\frac{4 a_{1} \rho^{4}}{\left(\varepsilon^{2}-\rho^{2}\right)_{+}^{2}} \\
\geq & 4 a_{1}\left[a_{1} \frac{(N-2)}{N}-1\right] \frac{\rho^{4}}{\left(\varepsilon^{2}-\rho^{2}\right)_{+}^{2}}+\frac{2(N-2) a_{2}}{\ln \rho^{2}} \\
& +\frac{2 a_{1}(N-4) \rho^{2}}{\left(\varepsilon^{2}-\rho^{2}\right)_{+}}+8 a_{1} a_{2} \frac{(N-2)}{N} \frac{\rho^{2}}{\left(\ln \rho^{2}\right)\left(\varepsilon^{2}-\rho^{2}\right)_{+}} .
\end{aligned}
$$

Choose

$$
a_{1}=\frac{2 N}{N-2}, \quad \varepsilon^{2}=2 e^{-a_{2} k},
$$

where $a_{2}, k>1$ will be chosen later. Then if $k a_{2}>\ln 4$

$$
\mathscr{H} \geq \frac{2(N-2) a_{2}}{\ln \rho^{2}}+4 a_{1} \frac{\rho^{2}}{\left(\varepsilon^{2}-\rho^{2}\right)_{+}}\left\{\frac{\rho^{2}}{\left(\varepsilon^{2}-\rho^{2}\right)_{+}}+\frac{N-4}{2}-\frac{4}{k} \frac{N-2}{N}\right\} .
$$

On the set $\mathscr{E}_{1} \equiv\left\{e^{-k a_{2}} \leq \rho^{2} \leq \varepsilon^{2}\right\}$, we have $\rho^{2} /\left(\varepsilon^{2}-\rho^{2}\right)_{+} \geq 1$ and

$$
\mathscr{H} \geq-\frac{4(N-2)}{k}+4 a_{1} \frac{\rho^{2}}{\left(\varepsilon^{2}-\rho^{2}\right)_{+}}\left\{\frac{N-2}{2}-\frac{2(N-2)}{N k}\right\} .
$$

Thus we may choose $k$ so large that

$$
\mathscr{H} \geq 0 \text { in } \mathscr{E}_{1} .
$$

On the set $\mathscr{E}_{2} \equiv\left\{\rho^{2}<e^{-k a_{2}}\right\}$, we have

$$
\mathscr{H} \geq \frac{2(N-2) a_{2}}{\ln \rho^{2}}-\frac{8 N}{N-2} \frac{\rho^{2}}{\left(\varepsilon^{2}-\rho^{2}\right)_{+}}\left[\frac{(4-N)_{+}}{2}+\frac{2(N-2)}{N k}\right] .
$$

Next if $Z=(1-h t)_{+} g$, by computation we have

$$
\begin{aligned}
\mathscr{L}(Z) & \equiv Z_{t}-\Delta Z^{m}=-h g-(1-h t)_{+}^{m} \frac{m g^{m}}{\rho^{2}} \mathscr{H} \\
& =g\left\{-h-(1-h t)_{+}^{m} \frac{m g^{m-1}}{\rho^{2}} \mathscr{H}\right\} .
\end{aligned}
$$


On $\mathscr{E}_{1}$ in view of $(\mathrm{C} .3), \mathscr{L}(Z) \leq 0$. On the set $\mathscr{E}_{2}$

$$
\begin{aligned}
& \mathscr{L}(Z) \leq g\left\{-h+\frac{m\left(\varepsilon^{2}-\rho^{2}\right)_{+}^{m-1}}{\rho^{N(m-1)+2}\left|\ln \rho^{2}\right|^{a_{2}(m-1)}}\right. \\
& \left.\times\left[\frac{2(N-2) a_{2}}{\left|\ln \rho^{2}\right|}+\frac{8 N}{N-2} \frac{\rho^{2}}{\left(\varepsilon^{2}-\rho^{2}\right)}\left(\frac{(4-N)_{+}}{2}+\frac{2(N-2)}{N k}\right)\right]\right\} .
\end{aligned}
$$

Since $N(m-1)+2=0$, we may select $a_{2}=N / 2$ to find $\mathscr{L}(Z) \leq g\{-h+C\}$ where $C$ is a constant depending only upon $N$ (and the choices of $a_{1}, a_{2}, k$ ). To prove the lemma we take $h=C$.

\section{APPENDIX D: ABOUT THE COMPARISON PRINCIPLE}

One of the main tools in the proof of the Harnack estimate is the comparison principle. Let $\varphi \geq 0$ be a smooth subsolution of (1.1) and consider the cylindrical domain

$$
Q \equiv \Omega_{*} \times\left(t_{1}, t_{2}\right), \quad \Omega_{*} \subset \Omega, \quad \partial \Omega_{*} \text { smooth }, \quad 0<t_{1}<t_{2} \leq T,
$$

whose parabolic boundary is $\Gamma \equiv \partial Q \cap\left\{t<t_{2}\right\}$. If $u \geq \varphi$ on $\Gamma$ then $u \geq \varphi$ in $Q$. This fact holds trivially if $u_{t} \in L_{\text {loc }}^{1}\left(\Omega_{T}\right)$ by $L^{1}$ techniques. In the weak formulation of

$$
(\partial / \partial t)(\varphi-u)_{+}-\Delta\left(\varphi^{m}-u^{m}\right)_{+} \leq 0 \text { in } Q,
$$

we take as testing function an approximation of $\operatorname{sign}\left(\varphi^{m}-u^{m}\right)_{+}$to yield, after standard calculations and limiting processes, $(\varphi-u)_{+} \equiv 0$ in $Q$.

If $u_{t} \notin L_{\text {loc }}^{1}\left(\Omega_{T}\right)$ we first observe that, since $D u^{m} \in L_{\text {loc }}^{2}\left(\Omega_{T}\right)$, and $u$ is locally Hölder continuous, $u$ is locally unique.

This can be seen by adapting the method of Oleinik-Kalashnikov-Yui-Lin Chzhou [19]. If $u, \bar{u}$ both solve (1.1) in $Q$ and $u=\bar{u}$ on $\Gamma$, then in the weak formulation of

$$
(\partial / \partial t)(u-\bar{u})-\Delta\left(u^{m}-\bar{u}^{m}\right)=0,
$$

we may take the testing function

$$
\eta=\int_{t}^{t^{*}}\left(u^{m}-\bar{u}^{m}\right)(\cdot, \tau) d \tau, \quad t_{1}<t<t^{*} \leq t_{2},
$$

to deduce $u\left(\cdot, t^{*}\right) \equiv \bar{u}\left(\cdot, t^{*}\right), \forall t_{1}<t<t^{*} \leq t_{2}$, (see [19] for details).

Then within $Q$, we work with the smooth approximations

$$
\begin{cases}\frac{\partial}{\partial t} u_{\varepsilon}-\Delta u_{\varepsilon}^{m}=0 & \text { in } Q, \\ \left.u_{\varepsilon}\right|_{\Gamma}=u+\varepsilon, & \varepsilon \in(0,1),\end{cases}
$$

and deduce $u_{\varepsilon} \geq \varphi$ in $Q, \forall \varepsilon \in(0,1)$. By the results of [6], $u_{\varepsilon} \in C^{\alpha}(\bar{Q})$ uniformly in $\varepsilon$, for some $\alpha \in(0,1)$, depending only upon $N, m$. Thus $u \geq \varphi$ in $Q$.

\section{REFERENCES}

1. D. Andreucci and E. DiBenedetto, $A$ new approach to initial traces in nonlinear filtration, Ann. Inst. H. Poincaré Anal. Non Linéaire 7 (1990), 305-334.

2. D. G. Aronson and J. Serrin, Local behaviour of solutions of quasilinear parabolic equations, Arch. Rational Mech. Anal. 25 (1967), 81-123. 
3. $\mathrm{Ph}$. Bénilan and $\mathrm{M}$. G. Crandall, The continuous dependence on $\phi$ of solutions of $u_{t}-$ $\Delta \phi(u)=0$, Indiana Univ. Math. J. 30 (1981), 161-177.

4. J. G. Berryman, Evolution of a stable profile for a class of nonlinear diffusion equations with fixed boundaries, J. Math. Phys. 18 (1977), 2108-2115.

5. J. G. Berryman and C. J. Holland, Stability of the separable solution for fast diffusion equation, Arch. Rational Mech. Anal. 74 (1980), 379-388.

6. Chen Ya-zhe and E. DiBenedetto, On the local behaviour of solutions of singular parabolic equations, Arch. Rational Mech. Anal. 103 (1988), 319-345.

7. E. DiBenedetto, Intrinsic Harnack type inequalities for solutions of certain degenerate parabolic equations, Arch. Rational Mech. Anal. 100 (1988), 129-147.

8. E. DiBenedetto and M. A. Herrero, Nonnegative solutions of the evolution p-Laplacian equation. Initial traces and Cauchy problem when $1<p<2$, Arch. Rational Mech. Anal. 111 (1990), 225-290.

9. J. Hadamard, Extension á l'équation de la chaleur d'un théorème de A. Harnack, Rend. Circ. Mat. Palermo 3 (1954), 337-346.

10. M. A. Herrero and M. Pierre, The Cauchy problem for $u_{t}=\Delta\left(u^{m}\right)$ when $0<m<1$, Trans. Amer. Math. Soc. 291 (1985), 145-158.

11. M. A. Herrero and J. L. Vazquez, Asymptotic behaviour of the solutions of a strongly non linear parabolic problem, Ann. Fac. Sci. Toulouse Math. 3 (1981), 113-127.

12. N. V. Krylov and M. V. Safonov, A certain property of solutions of parabolic equations with measurable coefficients, Math. USSR-Izv. 16 (1981), 151-164.

13. Y. C. Kwong, Interior and boundary regularity of solutions to a plasma problem, Proc. Amer. Math. Soc. 104 (1988), 472-478.

14. __ Asymptotic behaviour of a plasma type equation with finite extinction, Arch. Rational Mech. Anal. 104 (1988), 277-294.

15. __ Boundary behavior of solutions of the fast diffusion equation, Trans. Amer. Math. Soc. 322 (1990), 263-283.

16. O. A. Ladyzhenskaya, N. A. Solonnikov, and N. N. Ural'tseva, Linear and quasilinear equations of parabolic type, Transl. Math. Monographs, vol. 23, Amer. Math. Soc., Providence, R. I., 1968.

17. J. Moser, A Harnack inequality for parabolic differential equations, Comm. Pure Appl. Math. 17 (1964), 101-134.

18. H. Okuda and J. M. Dawson, Numerical simulation on plasma diffusion in three dimensions, Phys. Lett. 28 (1972), 1625-1629.

19. O. A. Oleinik, A. S. Kalashnikov, and Yui-Lin Chzhou, The Cauchy problem and boundary value problems for equations of the type of nonstationary filtration, Izv. Akad. Nauk SSSR Ser. Mat. 22 (1958), 667-704. (Russian)

20. M. Pierre, Nonlinear fast diffusion with measures as data, Proc. Nonlinear Parabolic Equations: Qualitative Properties of Solutions (Tesei and Boccardo, eds.), Pitman, New York, 1985.

21. B. Pini, Sulla soluzione generalizzata di Wiener per il primo problema di valori al contorno nel caso parabolico, Rend. Sem. Mat. Univ. Padova 23 (1954), 422-434.

22. J. Serrin, Local behaviour of solutions of quasilinear elliptic equations, Acta Math. 111 (1964), 101-134.

23. N. S. Trudinger, Pointwise estimates and quasilinear parabolic equations, Comm. Pure Appl. Math. 21 (1968), 205-226.

24. __ On Harnack type inequalities and their application to quasilinear elliptic partial differential equations, Comm. Pure Appl. Math. 20 (1967), 721-747.

Department of Mathematics, Northwestern University, Evanston, Illinois 60208

Department of Mathematics, Northern Illinois University, DeKalb, Illinois 60115 